\title{
Rayleigh-Taylor instability under a spherical substrate
}

\author{
Gioele Balestra, ${ }^{1}$ a) David Minh-Phuc Nguyen, ${ }^{1}$ and François Gallaire ${ }^{1}$ \\ Laboratory of Fluid Mechanics and Instabilities, EPFL, CH1015 Lausanne, \\ Switzerland
}

(Dated: 2 August 2018)

\begin{abstract}
We investigate the Rayleigh-Taylor instability of a thin viscous film coating the inside of a spherical substrate. The aim of this work is to find and characterize the instability pattern in this spherical geometry. In contrast to the Rayleigh-Taylor instability under a planar substrate, where the interface is asymptotically unstable with respect to infinitesimal perturbations, the drainage induced by the component of gravity tangent to a curved substrate stabilizes the liquid interface, making the system linearly asymptotically stable. By performing a linear optimal transient growth analysis we show that the double curvature of a spherical substrate yields a critical Bond number - prescribing the ratio between gravitational and capillary forces - before an initial growth of perturbations is possible two-times larger than for a circular cylindrical substrate. This linear transient growth analysis however does not yield any selection principle for an optimal azimuthal wavenumber and we have to resort to a fully nonlinear analysis. By numerically solving the nonlinear lubrication equation we find that the most amplified azimuthal wavenumber increases with the Bond number. Nonlinear interactions are responsible for the transfer of energy to higher-order harmonics. The larger the Bond number and the farther away from the apex of the sphere, the richer the wavenumber spectrum.
\end{abstract}

\section{INTRODUCTION}

The Rayleigh-Taylor instability (RTI) occurs when a fluid accelerates into another immiscible fluid ${ }^{1-3}$. In particular, the interface between the two fluids is unstable to the RTI when the heavier fluid lies on the lighter one and is accelerated by the gravitational field ${ }^{4}$. For example, a thin viscous film coating the underneath of a planar substrate is subjected to the $\mathrm{RTI}^{5,6}$. As observable in everyday life, condensed vapour under a bathroom ceiling or a kitchen lid forms isolated droplets rather than a uniform film. However, there are situations where a liquid film suspended on a fluid of lesser density is stable ${ }^{7}$. For instance, hollow chocolate eggs are fabricated by coating the interior of the mold which is then turned upside down to allow the excess chocolate to drain out, and yet, the liquid chocolate does not destabilize into droplets yielding an egg of nearly uniform thickness upon cooling ${ }^{8}$. The geometry of the substrate plays therefore a crucial role in the stabilization of the RTI through the modification of the base-state interfacial curvature ${ }^{9}$.

Controlling and characterizing the RTI is fundamental for many technical applications, ranging from the coating industry ${ }^{10}$, where the instability would result into nonuniform products or loss of material, to the plasma facing components in nuclear fusion reactors ${ }^{11,12}$, where liquid metals coating the inside of the tokamak are used to protect the walls from the high-temperature plasma. Furthermore, thin-film instabilities have been recently proposed as possible fabrication pathways to shape materials ${ }^{13}$.

In the canonical RTI, a thin-viscous fluid is coated underneath a horizontal substrate ${ }^{14}$. The interface is asymptotically unstable to disturbances having a wavelength larger than $2 \pi \ell_{c}^{*}$, where $\ell_{c}^{*}=\sqrt{\gamma /(\rho g)}$ is the capillary length with $\gamma$ the surface tension, $\rho$ the density and $g$ the gravitational acceleration. The linearly most amplified disturbance has a wavelength $\lambda_{\max }^{*}=2 \pi \sqrt{2} \ell_{c}^{*}$. In the absence of contact lines, the problem is isotropic and the

\footnotetext{
a) Electronic mail: gioele.balestra@epfl.ch
} 
dominant pattern has a hexagonal or an axial symmetry ${ }^{14}$. When the perturbations of the thin film are forced by the presence of contact lines at the boundaries, the interface first destabilizes into rolls, one-dimensional structures aligned with the boundaries and propagating into the domain ${ }^{15}$; a two-dimensional pattern of droplet eventually results from the nonlinear interactions. Fermigier et al. ${ }^{14}$ have shown that the nonlinear interactions promote a hexagonal pattern while weakening the growth of rolls. The resulting droplets might translate, bounce or collide and pinch-off for thick coatings ${ }^{16}$.

By adding one curvature to the substrate, i.e. considering a thin film coating the interior of a cylinder of radius $R$, Trinh et al. ${ }^{7}$ have shown that the interface is asymptotically stable with respect to infinitesimal perturbations and only an algebraic growth at short time can be achieved for sufficiently large Bond numbers $B o=H_{i}^{*} R / \ell_{c}^{* 2}$, where $H_{i}^{*}$ is the average initial film thickness. In a previous work of us, we have shown that the stabilization induced by the curvature has a twofold reason ${ }^{17}$. First, the gravity has not only a component orthogonal to the interface, promoting the instability, but also a component tangent to the interface, which is responsible for the drainage of the film. The film becomes thinner and thinner as time evolves, leaving only a short time for the instability to be effective. Second, the gravity-induced drainage stretches the perturbations which form at the top of the cylinder, further stabilizing their growth. Depending on the Bond number and the initial disturbance amplitude, the transient growth might be sufficiently large to trigger nonlinear effects resulting in the formation of droplets sliding along the cylinder walls or even droplets pinching-off from the substrate.

The cylindrical geometry of the substrate breaks the symmetry of the problem and the anisotropy of the forces acting on the thin film modifies the most amplified pattern ${ }^{18}$. At moderate Bond numbers, rivulets form rather than droplets. The thin film destabilizes into structures whose wavevector is aligned with the axis of the cylinder while its wavelength corresponds to the one of the classical RTI, $\lambda_{\max }^{*}{ }^{18}$. Such axial disturbances dominate over perturbations with a component in the polar direction as they experience only the thinning of the film, but are not further stabilized by the drainage-induced stretching. The flowanisotropy is the key to the stronger linear growth of rivulets. The latter resemble to the rolls of the classical RTI, yet with the difference that they persist over the whole time of the instability. The transition to a two-dimensional pattern of droplets relies solely on nonlinear interactions and occurs only for large Bond numbers ${ }^{18}$. Therefore, for a cylindrical substrate, the liquid film can either drain smoothly for Bond numbers smaller than the critical value $B o=12$, or it can destabilize into rivulets or a two-dimensional pattern of dripping droplets for moderate and large Bond numbers, respectively.

With the knowledge gained by the analysis of the RTI for a unidirectionally curved substrate, it can be already guessed that the key to the success of chocolatier in fabricating nearly uniform eggs results from the stabilizing effect induced by the substrate curvature on the RTI and the small Bond number value, i.e. the small radii. However, what would be the disturbance pattern at large $B o$ for a thin film underneath a spherical substrate where the curvature is again isotropic?

Here we undertake the stability analysis of the RTI of a thin film coating the interior of a sphere of radius $R$. Albeit the employed technique is similar to the one for a cylindrical substrate of Ref. ${ }^{18}$, we contrast our results with the ones of the cylindrical case. The linear stability analysis proves that the spherical geometry has a stabilizing effect which is twice as strong as the one for a cylindrical substrate. Furthermore, we show that given the spherical symmetry of the system, the linear optimal transient growth analysis does not select any particular azimuthal mode. Nonlinear numerical simulations of the governing equations allow us to show that there is an optimal azimuthal mode depending on the Bond number and on the disturbance amplitude. The nonlinear interactions are responsible for the transfer of energy from one mode to the other, creating an energy cascade in space and time, which is much richer than for the cylindrical substrate. In particular, the spherical geometry results in the widening of the perturbation spectrum as the distance from the pole increases, similarly as what is observed for an initially punctual perturbation for the classical RTI ${ }^{14}$. Nonetheless, the arising perturbation structures are intrinsically related 
to the spherical geometry. The nonlinear dynamics is characterized by introducing spatiotemporal diagrams for the disturbance norm along the azimuthal direction as well as for the azimuthal mode with the largest amplitude.

The governing equations are given in Sec. II A and specified for the region close to the north pole in Sec. II B. The linear optimal transient growth analysis is presented in Sec. III. In particular, the linear disturbance solution at the north pole is given in Sec. III A, followed by the optimal growth analysis in Sec. III B. The nonlinear results for the linear optimal initial conditions are described in Sec. IV, where the spatio-temporal diagrams are introduced in Sec. IV A. The effects of the initial azimuthal wavenumber and the Bond number are presented in Sec. IV B and Sec. IV C, respectively, whereas the sliding-droplet velocity is discussed in Sec. IV D. Finally, the results for the nonlinear evolutions of random noise initial conditions are discussed in Sec. V. Conclusions are drawn in Sec. VI.

\section{GOVERNING EQUATION}

\section{A. Entire upper hemisphere}

A film of initial average thickness $H_{i}^{*}$ coats the inside of a spherical substrate of inner radius $R$. Since the focus of this work is the destabilization of the interface under the action of the gravitational forces, only the upper hemisphere is relevant (see Fig. 1). The lower hemisphere would be relevant if one would investigate the destabilization of a thin film coating the outside of a spherical substrate. Given the small film thickness $H_{i}^{*}$ compared to

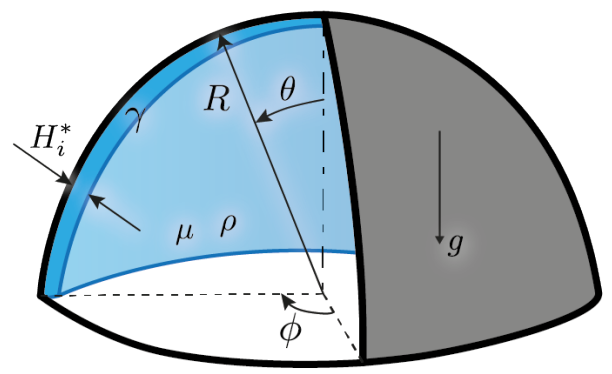

FIG. 1: Sketch of the problem geometry.

the sphere radius $R$, the aspect ratio is small, $\delta=H_{i}^{*} / R \ll 1$. Furthermore, we assume that inertial effects can be neglected, i.e. $R e \delta \ll 1$, where $R e=g \rho^{2} H_{i}^{* 3} /\left(3 \mu^{2}\right)$ is the Reynolds number. A lubrication approach ${ }^{19}$ can be employed to derive the governing equation for the film thickness. The characteristic time is the drainage time $\tau_{d}$, which is given by the ratio of the viscous over the gravitational stresses, $\tau_{d}=\mu R /\left(\rho g H_{i}^{* 2}\right)$, where $\mu$ is the dynamic viscosity, $\rho$ is the density, and $g$ is the gravitational field. By using the local mass conservation in spherical coordinates as well as $H_{i}^{*}$ and $\tau_{d}$ as length and time gauges of the problem, the evolution equation of the film thickness $\bar{H}(\theta, \phi, t)$ is (see Appendix A for the detailed derivation)

$\bar{H}_{t}+\frac{1}{3 \sin \theta}\left[\bar{H}^{3} \sin \theta(\underbrace{\frac{1}{B o} \bar{\kappa}_{\theta}}_{\mathrm{I}}+\underbrace{\delta \bar{H}_{\theta} \cos \theta}_{\mathrm{II}}+\underbrace{\sin \theta}_{\mathrm{III}})\right]_{\theta}+\frac{1}{3 \sin \theta}\left[\frac{\bar{H}^{3}}{\sin \theta}(\underbrace{\frac{1}{B o} \bar{\kappa}_{\phi}}_{\mathrm{I}}+\underbrace{\delta \bar{H}_{\phi} \cos \theta}_{\mathrm{II}})\right]_{\phi}=0$, 
where the capillary pressure gradient corresponds to the terms $\mathbf{I}$, the variation of the hydrostatic to the terms II and the drainage to the term III; indices represent partial derivatives. The modified Bond number, prescribing the ratio between gravitational and capillary forces, is given by $B o=\rho g H_{i}^{*} R / \gamma$, where $\gamma$ is the surface tension. The curvature $\bar{\kappa}$ up to the second order in $\delta$ is :

$$
\bar{\kappa}=2 \delta+\delta^{2}\left(\bar{H}_{\theta \theta}+\cot \theta \bar{H}_{\theta}+\csc ^{2} \theta \bar{H}_{\phi \phi}+2 \bar{H}\right)+O\left(\delta^{3}\right) .
$$

\section{B. Limit for the vicinity of the north pole}

Following previous studies ${ }^{7,17}$, the stability properties of the system are well captured by the analysis at the north pole. The lubrication equation (1) can be simplified when considering the region close to the north pole, i.e. $\theta \ll 1$. The change of variables $\theta=\delta^{1 / 2} r$ can be employed ${ }^{7}$ when $\delta \ll 1$. The curvature becomes

$$
\bar{\kappa}=\delta\left[2+\frac{1}{r} \frac{\partial}{\partial r}\left(r \bar{H}_{r}\right)+\frac{1}{r^{2}} \bar{H}_{\phi \phi}\right]+O\left(\delta^{2}\right)=\delta\left[2+\nabla^{2} \bar{H}\right]+O\left(\delta^{2}\right)=\delta \overline{\tilde{\kappa}}+O\left(\delta^{2}\right),
$$

where $\nabla^{2}$ is the Laplace operator in polar coordinates and $\overline{\tilde{\kappa}}=2+\nabla^{2} \bar{H}$ is the rescaled curvature. The mean curvature is indeed the double of the one of a cylinder. Using $\cos \theta \approx 1$ and $\sin \theta \approx \theta=\delta^{1 / 2} r$, equation (1) eventually reads:

$$
\bar{H}_{t}+\frac{1}{3 r}\left[\bar{H}^{3} r\left(\frac{1}{B o} \overline{\tilde{\kappa}}_{r}+\bar{H}_{r}+r\right)\right]_{r}+\frac{1}{3 r}\left[\frac{\bar{H}^{3}}{r}\left(\frac{1}{B o} \overline{\tilde{\kappa}}_{\phi}+\bar{H}_{\phi}\right)\right]_{\phi}=0 .
$$

The resulting equation is aspect-ratio independent and the Bond number is the only remaining parameter. Using the above mentioned change of variable and the small aspect ratio limit allows to transform the lubrication equation from a spherical coordinate system to a polar coordinate system in the vicinity of the north pole.

\section{LINEAR OPTIMAL TRANSIENT GROWTH ANALYSIS AT THE NORTH POLE}

\section{A. Linear disturbance solution}

Assuming an initial film thickness uniform in space, $\bar{H}(r, \phi, t)=1$, the lubrication equation in the vicinity of the north pole, Eq. (4), has an analytical solution, called drainage solution, given by

$$
H(T)=\frac{1}{\sqrt{T}},
$$

with $T=1+(4 / 3) t$ the rescaled time ${ }^{7,8,20}$ (see Appendix B for the derivation).

The film thickness is decomposed into this spatially uniform drainage solution $H$ and small space-dependent disturbances $\varepsilon h(r, \phi, T)$ :

$$
\bar{H}(r, \phi, T)=H(T)+\varepsilon h(r, \phi, T) \quad, \quad \varepsilon \ll 1 .
$$

The linear equation is obtained by entering the decomposition (6) into the lubrication equation (4) and collecting the terms at order $\varepsilon$ :

$$
h_{T}+\frac{1}{4 T^{3 / 2}}\left(\frac{\nabla^{4} h}{B o}+\nabla^{2} h\right)+\frac{3}{4 T}\left(r h_{r}+2 h\right)=0,
$$

where $\nabla^{2}$ and $\nabla^{4}$ are the Laplacian and bi-Laplacian in polar coordinates, respectively. The base flow $H$ is explicitly accounted for by the powers of $T$. 
The solution of equation (7) can be expressed in the eigenfunction basis of the Laplace operator in polar coordinates. Only the Bessel functions of the first kind, $J_{m_{0}}$, are not diverging neither at the origin nor at $r \rightarrow \infty$. Given the orthogonality of the eigenfunctions $J_{m_{0}}$ and following Parseval's theorem, we can consider mode per mode separately. For an initial condition of the form $h(r, \phi, 1)=h_{0}(r, \phi)=J_{m_{0}}(k r) \exp \left(i m_{0} \phi\right)+c . c$. , the solution is given by

$$
h(r, \phi, T)=A\left(k, m_{0}, T\right) J_{m_{0}}\left(k \frac{r}{T^{3 / 4}}\right) \exp \left(i m_{0} \phi\right)+c . c .,
$$

where the apparent radial wavenumber $k / T^{3 / 4}$ is time-dependent as for the flow inside a cylinder ${ }^{7,18}$. The amplitude $A$ satisfies:

$$
A_{T}+\frac{1}{4}\left(\frac{1}{B o} \frac{k^{4}}{T^{9 / 2}}-\frac{k^{2}}{T^{3}}+\frac{6}{T}\right) A=0,
$$

which is obtained by entering Eq. (8) into the linear disturbance equation (7). The solution of (9) reads

$$
A\left(k, m_{0}, T\right)=A(k, T)=\frac{1}{T^{3 / 2}} \exp \left[\left(1-\frac{1}{T^{2}}\right) \frac{k^{2}}{8}-\frac{1}{B o}\left(1-\frac{1}{T^{7 / 2}}\right) \frac{k^{4}}{14}\right] .
$$

It has to be observed that the amplitude (10) does not depend on the azimuthal number $m_{0}$.

The exponential term in Eq. (10) results from the Rayleigh-Taylor instability, where both the destabilizing term in $k^{2}$ and the stabilizing term in $k^{4}$ can be evidenced. The timedependent coefficients of the destabilizing and stabilizing terms saturate for large times due to the thinning of the film. Thus, the largest value of the exponential term only depends on the wavenumber $k$ and on the Bond number. Furthermore, the gravity-induced drainage also enters through the algebraic term $T^{-3 / 2}$, which, together with the saturated exponential term, is responsible for the linear asymptotic stability of the system, $\lim _{T \rightarrow \infty} A(k, T, B o)=$ 0 . Hence, the disturbances can grow only over a finite, transient time.

The initial amplitude evolution is given by

$$
A(k, T \rightarrow 1) \sim 1+\frac{\left(k^{2}-6\right) B o-k^{4}}{4 B o}(T-1)+O[T-1]^{2} .
$$

Initial growth only occurs if $k^{2}-6-k^{4} / B o>0$. This condition is best met for

$$
k_{0}=\sqrt{\frac{B o}{2}},
$$

which is the initially most amplified wavenumber, corresponding to the wavenumber with the largest linear growth in the planar Rayleigh-Taylor instability ${ }^{14}$. Initial growth is only possible for $B o>24$, which is the double of the critical Bond number for a cylindrical geometry $^{7,17}$. This result is imputable to the fact that the drainage occurs over the two principal radii of curvature of the sphere and not only the unique one of the cylinder. As observable in Eq. (5), the film thins two times as fast as for a cylinder ${ }^{20}$. During the reviewing process, one of the referee pointed out that this result was already described in Hammoud $^{21}$, work we were not aware of.

\section{B. Optimal growth}

As visible in Fig. 2, the amplitude of the disturbances for a specified initial wavenumber presents a maximum at a given time; both amplitude and time increase with the value of the Bond number. 


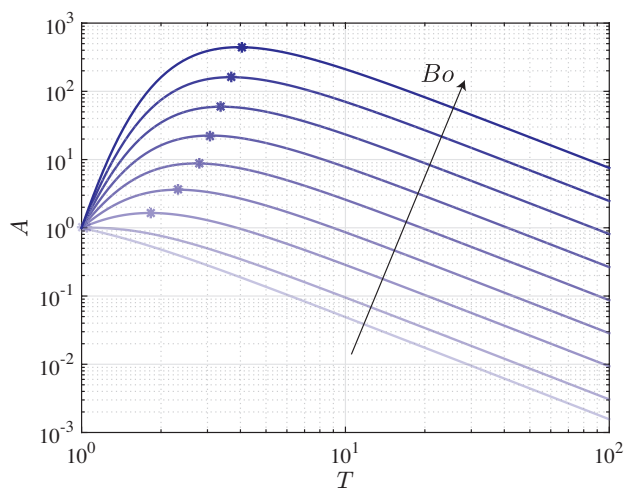

FIG. 2: Temporal evolution of the linear amplitude $A$ for different Bond numbers, $B o=10,25,50,75,100,125,150,175$ and 200, with the initial optimal wavenumber $k_{0}$. Asterisks denote the largest amplitudes.

The amplitude $A$ of the disturbances can be optimized as a function of the wavenumber $k$ and time $T$, which will become functions of the Bond number. The optimal wavenumber $k_{\max }$ is given by

$$
\frac{\partial A}{\partial k}=0 \Leftrightarrow k_{\max }(T)=\sqrt{\frac{7}{8} \frac{\left(1+T+T^{7 / 2}+T^{9 / 2}\right) T^{3 / 2}}{\left(1+T+T^{2}+T^{3}+T^{4}+T^{5}+T^{6}\right)} B o},
$$

whose limits are $\lim _{T \rightarrow 1} k_{\max }(T)=\sqrt{B o / 2}$ and $\lim _{T \rightarrow \infty} k_{\max }(T)=\sqrt{7 B o / 8}$ (see inset of Fig. 3(a)) . The largest growth reached with $k_{\max }(T)$ is thus given by

$$
A_{k_{\max }}(T)=A\left(k_{\max }, T\right)=\frac{1}{T^{3 / 2}} \exp \left[\frac{7\left(T^{1 / 2}-1\right)\left(T^{1 / 2}+1\right)^{2}(T+1)^{2} B o}{128\left(T^{1 / 2}+T+T^{3 / 2}+T^{2}+T^{5 / 2}+T^{3}+T^{7 / 2}\right)}\right] .
$$

Solving $\partial A_{k_{\max }} / \partial T=0$, the optimal time $T_{A_{\max }}$ is found to be a monotonic function of the Bond number, approximated by the empirical fitting law $T_{A_{\max }}=0.8363 B o^{0.4}-1.846$, which holds for $24<B o \lesssim 300$ (see Fig. 3(a)).

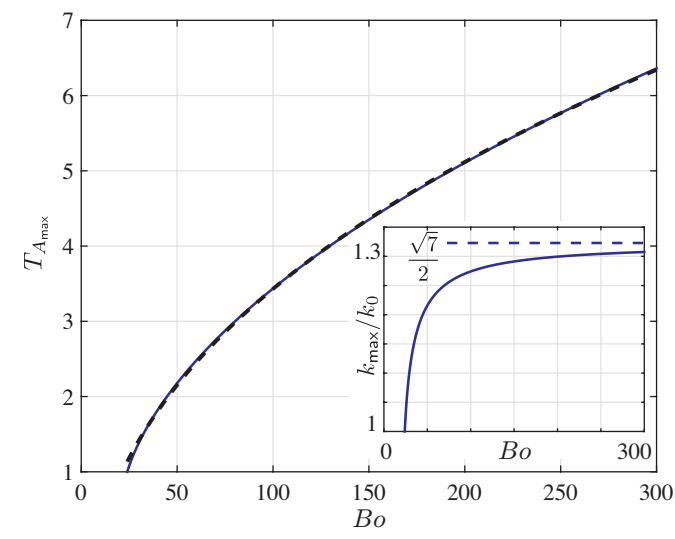

(a)

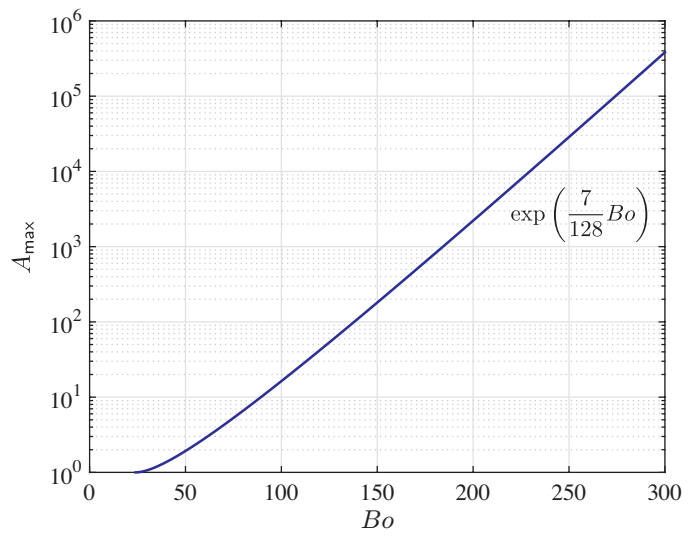

(b)

FIG. 3: (a) Optimal time $T_{A_{\max }}$ (blue solid line), with its approximation (black dashed line), and wavenumber $k_{\max }$ (inset) giving the largest amplitude $A_{\max }$ (b) as a function of the Bond number.

With the optimal wavenumber $k_{\max }$ and the optimal time $T_{A_{\max }}$, one obtains the largest 
possible amplitude $A_{\max }$ (see Fig. 3(b)). For $B o \gg 24$, the largest optimal amplitude is found to be an exponential function of the Bond number, $A_{\max } \sim \exp (7 B o / 128)$.

By performing the linear optimal transient growth analysis we have found the optimal wavenumber in the radial direction and the optimal time, but we cannot conclude on the optimal azimuthal wavenumber, reason why a nonlinear study is undertaken next.

\section{NONLINEAR EVOLUTION OF THE LINEAR OPTIMAL INITIAL CONDITIONS}

Equipped with the insights of the linear optimal transient growth analysis in the vicinity of the north pole, we investigate hereafter the nonlinear evolution of the perturbations over the entire geometry. The nonlinear lubrication equation (1) for the total thickness $\bar{H}$ is solved numerically for $\theta \in[0, \pi]$ and $\phi \in[0,2 \pi]$ (see Appendix $\mathrm{C}$ for details on the numerical method). Periodic boundary conditions are used for the azimuthal direction $\phi$ and no flux is imposed at the poles $\theta=0$ and $\theta=\pi$. Since we are interested in the unstable region of the film coated under the spherical geometry, only the dynamics for the upper hemisphere is presented. In the lower hemisphere the liquid accumulates in a way similar to what is found for a circular geometry ${ }^{17}$. The initial condition for the film thickness is given by

$$
\bar{H}_{0}(\theta, \phi)=1+\varepsilon J_{m_{0}}\left(k_{\max } \theta\right) \cos \left(m_{0} \phi\right),
$$

where $k_{\max }$ is the optimal wavenumber given by equation (13) and $m_{0}$ is the azimuthal wavenumber. To avoid the formation of a singular droplet at the pole, the axisymmetric perturbation $m_{0}=0$ is not considered. Due to the lubrication approach pursued in this study, droplets pinching off from the substrate ${ }^{22,23}$ cannot be taken into account.

The linear film thickness evolution predicted by the analytical solution (8), together with its amplitude, Eq. (10), can be compared to the film thickness given by the nonlinear evolution of the fundamental mode over the entire geometry. As visible in Fig. 4, the

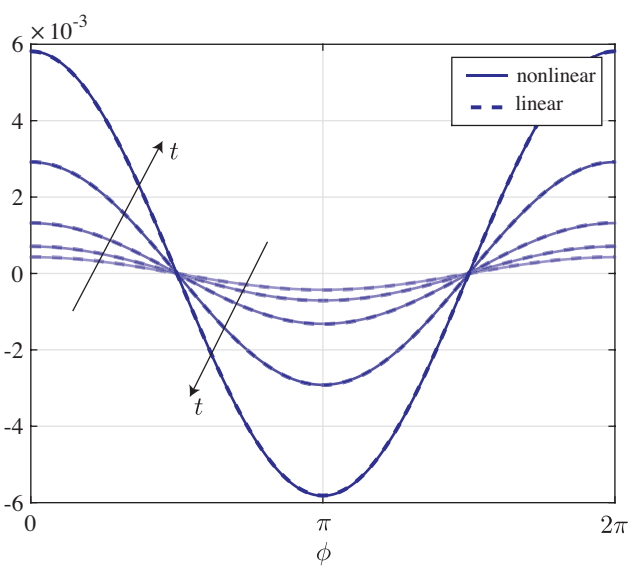

(a)

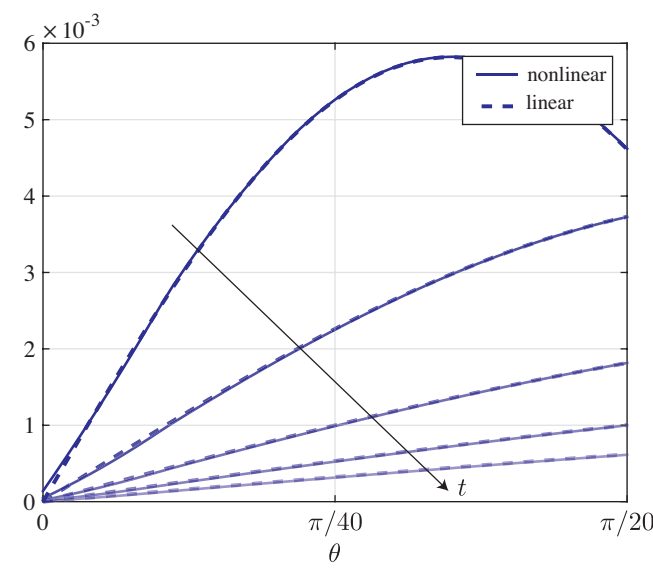

(b)

FIG. 4: Comparison between the linear evolution of the film thickness given by equation

(8) (dashed line) and the one of the fundamental (solid line) obtained by the nonlinear evolution of the initial condition (15) with $B o=25, m_{0}=1$ and $\varepsilon=A(T=1)=10^{-2}$ at the times $t=0,1,2,3$ and 4 . (a) Film thickness along the polar direction at $\theta=\pi / 30$ for $\phi \in[0,2 \pi]$ and (b) along the azimuthal direction for $\theta \in[0, \pi / 20]$ and $\phi=0$. The agreement remains good also for later times.

analytical and numerical solutions are in very good agreement. Hence, the initial evolution is linear and the analytical solution for the region close to the north pole is correct also for finite, yet small, polar angles. The larger the Bond number, the earlier the nonlinear 
evolution will differ from the linear prediction due to the nonlinear interactions, as we will discuss in the following.

To illustrate the typical nonlinear dynamics of the RTI, the total film thickness over the upper hemisphere at successive time instants is shown in Fig. 5 for $B o=150, \varepsilon=10^{-2}$ and $m_{0}=6$ (see also Supplementary Movie $1^{24}$ ). The six largest initial perturbation peaks
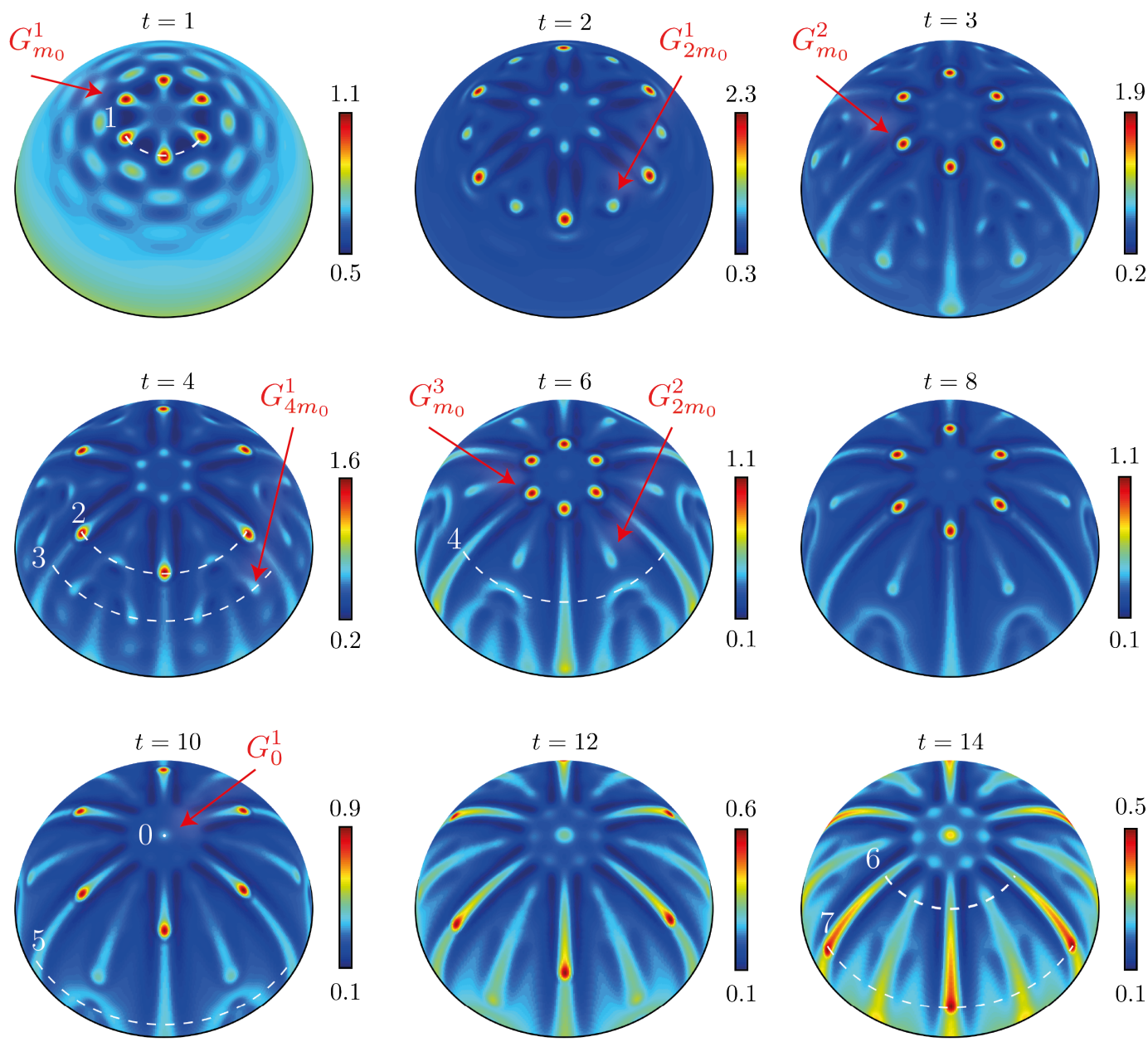

FIG. 5: Nonlinear evolution of the film thickness for the initial condition given by equation (15) for $B o=150, m_{0}=6$ and $\varepsilon=10^{-2}$ at the times $t=1,2,3,4,6,8,10,12$ and 14. The film thickness is indicated by the colorbar, which changes scaled nonmonotonically with time. The evolution with a constant colorbar is shown in Fig. 22 in the Appendix D. Particular spatial locations are indicated by a white dashed line; the numbers are used also in the following figures. The droplet generations $G_{m_{0}}, G_{2 m_{0}}, G_{2 m_{0}}$ and $G_{0}$ are also highlighted. See Supplementary Movie $1^{24}$ for the entire evolution.

rapidly grow into droplets $(t=1$, location 1$)$. This first generation of droplets, denoted as $G_{m_{0}}^{1}$, quickly slides along the spherical substrate, catching up the second largest initial perturbations, denoted as $G_{2 m_{0}}^{1}(t=2)$. Subsequently, a second generation of six droplets, $G_{m_{0}}^{2}$, forms close to the pole $(t=3)$. The droplets $G_{m_{0}}^{2}$ translate more easily due to the thicker film left by the former generation of droplets $G_{m_{0}}^{1}$ along their trajectory, which reduces the viscous drag $(t=4$, location 2), thereby flowing in the wakes of their predecessor. The droplet velocity will be quantified later in Sec. IV C. In the mean time, the nonlinear interactions promote the formation of additional droplets, $G_{4 m_{0}}^{1}(t=4$, location 3$)$. When draining between the wakes of previous droplets, these additional perturbations form an 
inverted-U shape perturbation, whose uppermost peak $(t=6$, location 4$)$ is followed by a second generation of droplets $G_{2 m_{0}}^{2}$, as well as by a third generation $G_{m_{0}}^{3}$. This peculiar inverted-U shaped structure is swept away by the draining flow $(t=10$, location 5$)$, leaving only the wakes of the fundamental droplets $G_{m_{0}}^{1,2,3}$ and the secondary droplets $G_{2 m_{0}}^{1,2}$. As time increases, a single droplet $G_{0}^{1}$ forms at the north pole $(t=10$, location 0$)$. Eventually, after the three generations of fundamental droplets and the two of secondary droplets are swept away, the thin film is only perturbed along their wakes $(t=14$, locations 6$)$ as well as along additional regions between the wakes for large polar angles (see location 7 ) and at the pole. The wake perturbations at late times are extended over a large range of polar angles, excluding the pole, and correspond to rivulets rather than to localized droplets.

Hereafter, the different stages of the nonlinear evolution of the RTI under a spherical substrate are discussed and characterized in more detail.

\section{A. Spatio-temporal amplitude diagrams}

The nonlinear film thickness perturbation is given by

$$
\varepsilon h(\theta, \phi, t)=\bar{H}(\theta, \phi, t)-H(\theta, \phi, t) .
$$

The drainage solution over the entire geometry $H(\theta, \phi, t)$ is obtained numerically by solving equation (1) with a smooth initial condition $H_{0}(\theta, \phi)=1$, similarly to what done in Ref. ${ }^{17}$. Although the Bond number might be larger than the critical value for transient growth, the accuracy of the numerical scheme is good enough to avoid numerical errors to be amplified. The obtained solution remains smooth and can be considered as the drainage solution (see Appendix C for further details).

Given the periodicity of the disturbances in the azimuthal direction, it is natural to define a disturbance norm over the azimuthal direction

$$
\mathcal{H}(\theta, t)=\left(\int_{0}^{2 \pi}|\varepsilon h(\theta, \phi, t)|^{2} d \phi\right)^{1 / 2}
$$

and to study its spatio-temporal dynamics. The result for the evolution presented in Fig. 5 is shown in the diagram of Fig. 6. The main stages of the previous qualitative description of the perturbation dynamics can be highlighted in the spatio-temporal diagram. The fast motion of the first generation $G_{m_{0}}^{1}$ of droplets can be seen by the largest-amplitude ridge (location 1), along which the largest disturbance amplitude is found (red star). The droplets of the first generation catch up the other smaller perturbations, represented by the

thin rays at short times. The successive droplet generations $G_{m_{0}}^{2}$ and $G_{m_{0}}^{3}$ correspond to the other large-amplitude ridges (on which points 2 and 7 are located). It can observed that the sliding velocity of the successive droplet generations is slower and slower, as the film becomes thinner due to the drainage. Finally, the amplitude close to $\theta=0$ increases with time due to the droplet formation at the north pole.

The evolution of the disturbance amplitude at the pole is shown in the inset of Fig. 6 . The disturbance is initially vanishing, since for $m_{0}=6$ there is no initial perturbation at $\theta=0$. However, due to nonlinear interactions, a droplet forms at the pole and its initial growth is exponential with the Rayleigh-Taylor growth rate $H_{f}^{3}\left(k_{f}^{2}-k_{f}^{4} / B o\right) / 3^{14}$, where the film thickness $H_{f}$ corresponds to the mean film thickness at the instant of formation, here $t_{f} \simeq 8$, and $k_{f}$ is the optimal wavenumber given by Eq. (13) evaluated at $t_{f}$. Note that after $t>20$ the growth is no longer exponential due to nonlinear effects.

To further unravel the mechanisms at play in the nonlinear evolution of the perturbations it is useful to study the amplitude of the azimuthal modes obtained by Fourier transform of the perturbations along $\phi$. The thickness perturbation can be decomposed into the Fourier series

$$
\varepsilon h(\theta, \phi, t)=\sum_{m \in \mathbb{N}} A_{m}(\theta, t) \exp (i m \phi)+c . c .,
$$




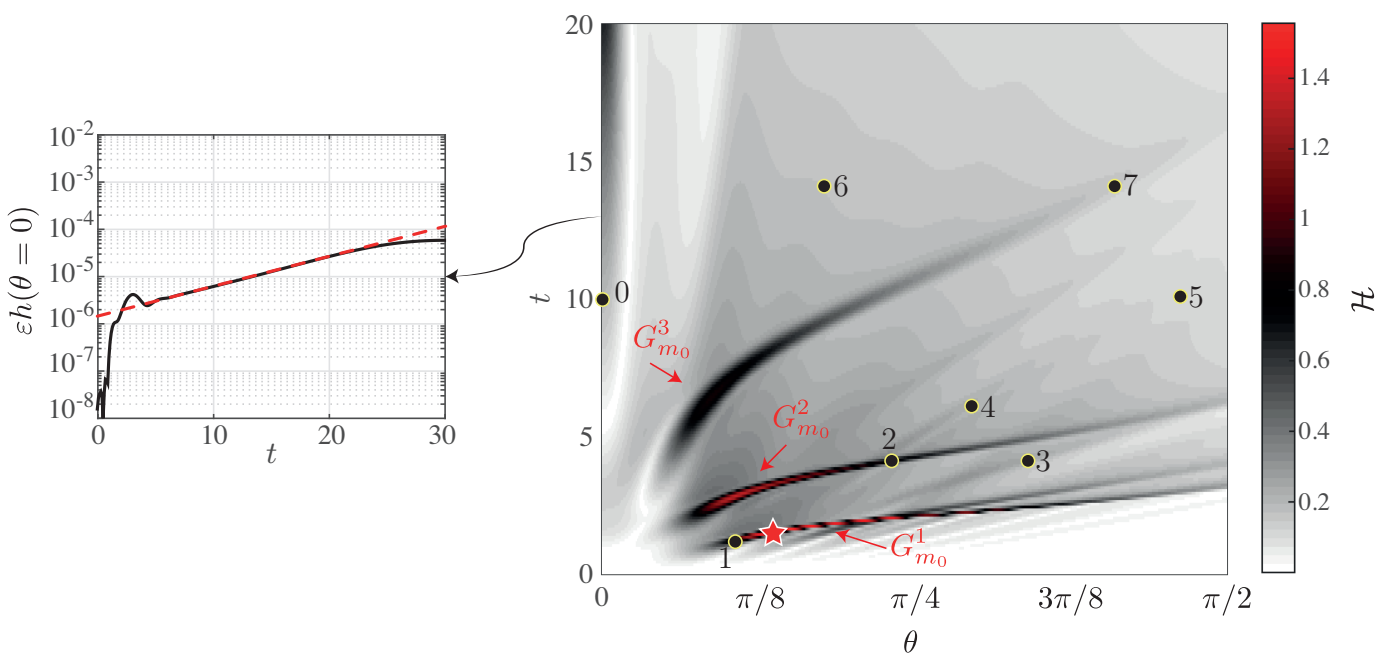

FIG. 6: Azimuthal disturbance norm $\mathcal{H}$ for the evolution shown in Fig. 5, namely for $B o=150, m_{0}=6$ and $\varepsilon=10^{-2}$ with a linear optimal initial condition. The numbered points correspond to the locations specified in Fig. 5 and the red star highlights the instant of largest amplitude. The amplitude ridges of the droplets generations $G_{m_{0}}^{1,2,3}$ are

also indicated. The discontinuous appearance of the amplitude is due to the limited temporal sampling. The film thickness disturbance amplitude at the pole, $\varepsilon h(\theta=0)$, is shown in the inset on the left (black solid line), together with the growth predicted by the linear theory (red dashed line).

where $A_{m}(\theta, t)$ is the space and time dependent amplitude of the azimuthal modes $m$. The spatio-temporal diagrams for the amplitudes of the fundamental $A_{m_{0}}$ and the harmonics $A_{2 m_{0}}, A_{4 m_{0}}$ and $A_{0}$ are presented in Fig. 7 . The modes with the largest amplitude are the fundamental $m_{0}$ and the first harmonic $2 m_{0}$, as already observed in the snapshot for $t=14$ in Fig. 5. All the modes have a similar spatio-temporal dynamics, with a finite number of ridges corresponding to the number of droplet generations. However, the higher the harmonics, the larger the polar angle $\theta$ where they appear (see also Fig. $5, t=14$ ). The axisymmetric perturbation $m=0$ clearly dominates at very small angles, close to the north pole, where a stationary droplet forms. It has to be stressed that the mode $m=0$ does not only correspond to a droplet at the pole, but also to any axisymmetric perturbation resulting from a modification of the mean film thickness through nonlinear interactions compared to the drainage solution. A similar effect has been found for the flow inside a cylinder ${ }^{18}$. Therefore, away from the pole, the mode $m=0$ indicating a mean flow modification is not relevant for the prediction of the instability pattern.

With the use of Fig. 7 we infer that location 3 corresponds to the second harmonics $4 m_{0}$, whereas the location 4 is mainly composed by the first harmonic $2 m_{0}$, together with the fundamental $m_{0}$. The mode with the largest amplitude at the location 6 is $2 m_{0}$, whereas at location 7 it is $4 m_{0}$.

The perturbation can be characterized by the azimuthal mode with the largest amplitude at each time $t$ and polar angle $\theta$, excluding the mode $m=0$ in light of the previous remark. The observations of Fig. 7 are confirmed by the spatio-temporal diagram of Fig. 8 . Starting with $m_{0}=6$, the nonlinear interactions excite the modes $2 m_{0}=12,3 m_{0}=18$ and $4 m_{0}=24$. At late times, the harmonics $2 m_{0}$ and $4 m_{0}$ are dominant for angles greater than $\theta_{1}$ and $\theta_{2}$, respectively (see Fig. 8).

Furthermore, with the help of the schematic of Fig. 9(a), it can be also inferred that the perturbation at location 5 corresponds to a mode with $m=3 m_{0}$. The ray in Fig. 8 where locations 4 and 5 are situated corresponds to the drainage of the inverted-U shape perturbations. These perturbations disappear for $t \gtrsim 14$, leaving only the first and sec- 


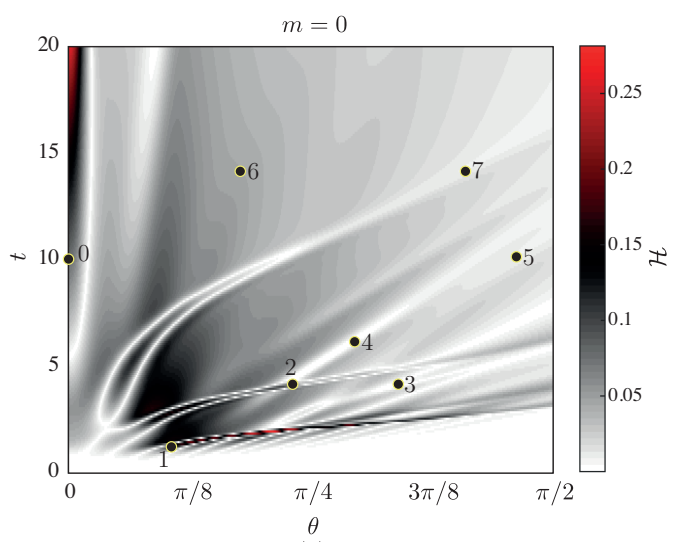

(a)

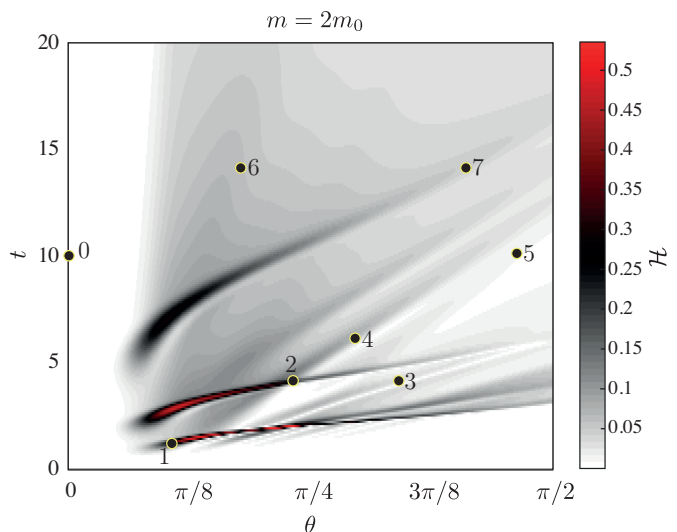

(c)

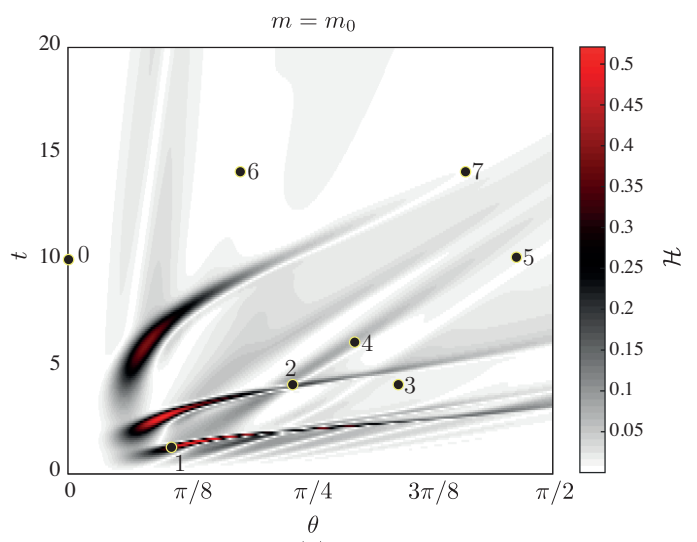

(b)

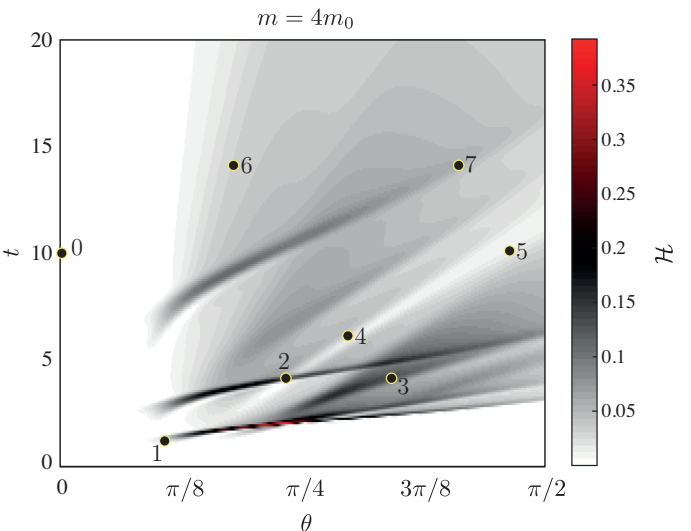

(d)

FIG. 7: Amplitude of the azimuthal modes $m=0$ (a), $m=m_{0}$ (b), $m=2 m_{0}$ (c) and $m=4 m_{0}$ (d) for $B o=150, m_{0}=6$ and $\varepsilon=10^{-2}$ with a linear optimal initial condition. The numbered points correspond to the locations specified in Fig. 5.

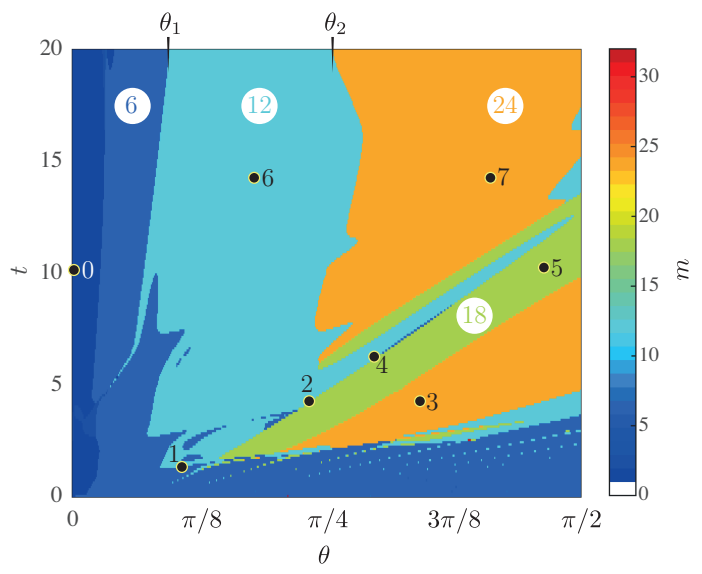

FIG. 8: Azimuthal mode $m$ with the largest amplitude for $B o=150, m_{0}=6$ and $\varepsilon=10^{-2}$ with a linear optimal initial condition. The colorbar indicates the $m$-values.

Numbered points correspond to the locations in Fig. 5 and numbers on a white background correspond to the $m$-value. $\theta_{1}$ and $\theta_{2}$ indicate the polar angle at $t=20$ where the largest mode switches to the higher harmonics. 


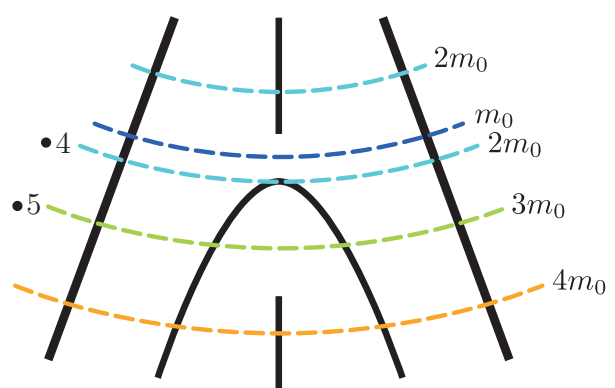

(a)

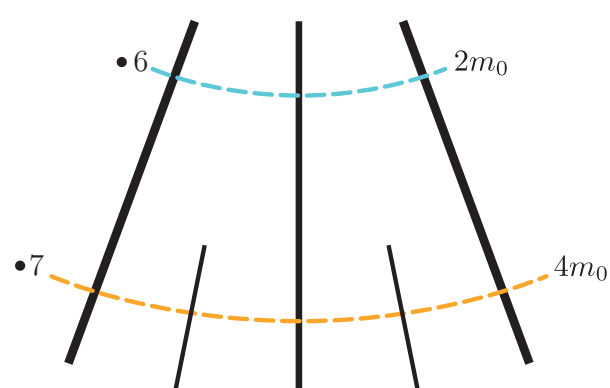

(b)

FIG. 9: Schematic representation of (a) the inverted-U pattern and (b) the late time pattern, with the corresponding locations in Fig. 8 (left) and the wavenumbers (right).

ond harmonics of the perturbations, locations 6 and 7 , depending on the polar angle (see Fig. 9(b)).

In order to highlight the nonlinear transfer of energy from one azimuthal mode to the higher harmonics, we define the disturbance-energy norm over the entire upper hemisphere for the azimuthal mode $m$ as

$$
\mathcal{E}_{m}(t)=\left(\int_{0}^{\pi / 2}\left|A_{m}(\theta, t)\right|^{2} d \theta\right)^{1 / 2} .
$$

The evolution of $\mathcal{E}_{m}$ for the considered case is presented in Fig. 10. The azimuthal mode

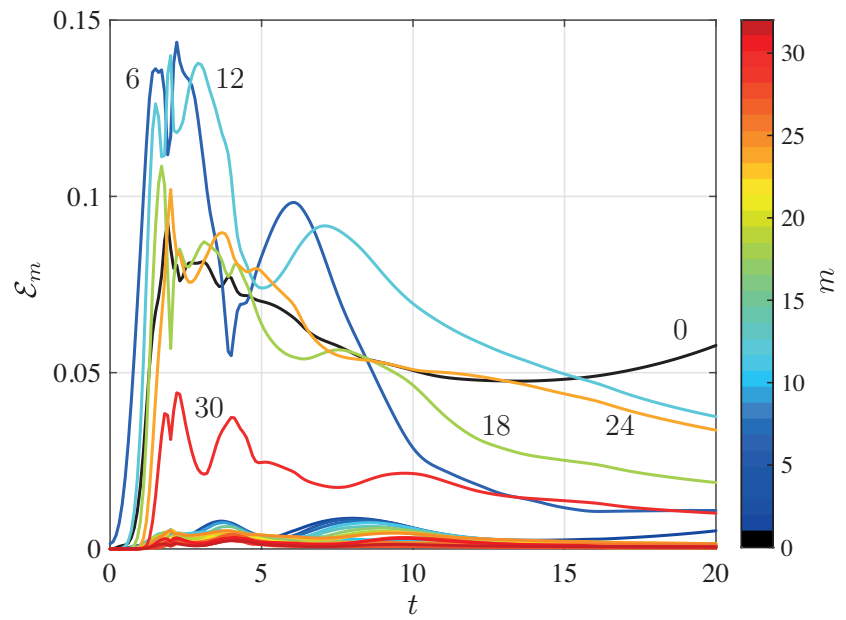

FIG. 10: Evolution of the energy norm $\mathcal{E}_{m}$ of the different modes over the upper hemisphere for $B o=150, m_{0}=6$ and $\varepsilon=10^{-2}$ with a linear optimal initial condition. The colorbar indicates the $m$-values, also highlighted on the plot for the dominant modes.

with the largest amplitude is the fundamental $m=6$, followed by the harmonics $m=12$, $m=18$ and $m=24$. The mode $m=30$ reaches also a relatively large amplitude. Therefore, the nonlinear interactions are responsible for the transfer of energy from one harmonics to the higher ones. Furthermore, the number of peaks in the disturbance energy for a given azimuthal mode corresponds to the number of droplet generations formed. For instance, for $m=6$ one observes three peaks corresponding to the three droplet generations of Fig. 5 . Note also that the mode $m=0$ grows at large times due to the droplet at the pole, whereas all the other modes decay in time. 
Thus far the analysis has hinged on the particular case $B o=150, m_{0}=6$ and $\varepsilon=10^{-2}$, which allowed us to introduce the different quantities necessary to quantify the nonlinear dynamics. In the following sections, the effects of the fundamental azimuthal wavenumber and of the Bond number are investigated.

\section{B. Effect of the initial azimuthal wavenumber}

The film thickness maps at $t=4$ for the initial azimuthal wavenumbers $m_{0}=1,6$ and 12 are shown in Fig. 11. Focusing for now on the second row for $B o=150$, we see that for $m_{0}=1$, when initially only one peak is present in the disturbance field, the resulting droplet generates a cascade of several droplets when sliding on the substrate. The pattern is symmetric with respect to the wake of the first droplet, but the appearance of a dominating azimuthal wavelength is less clear. For $m_{0}=12$, instead, the pattern of equally spaced wake-rivulets is already well established.

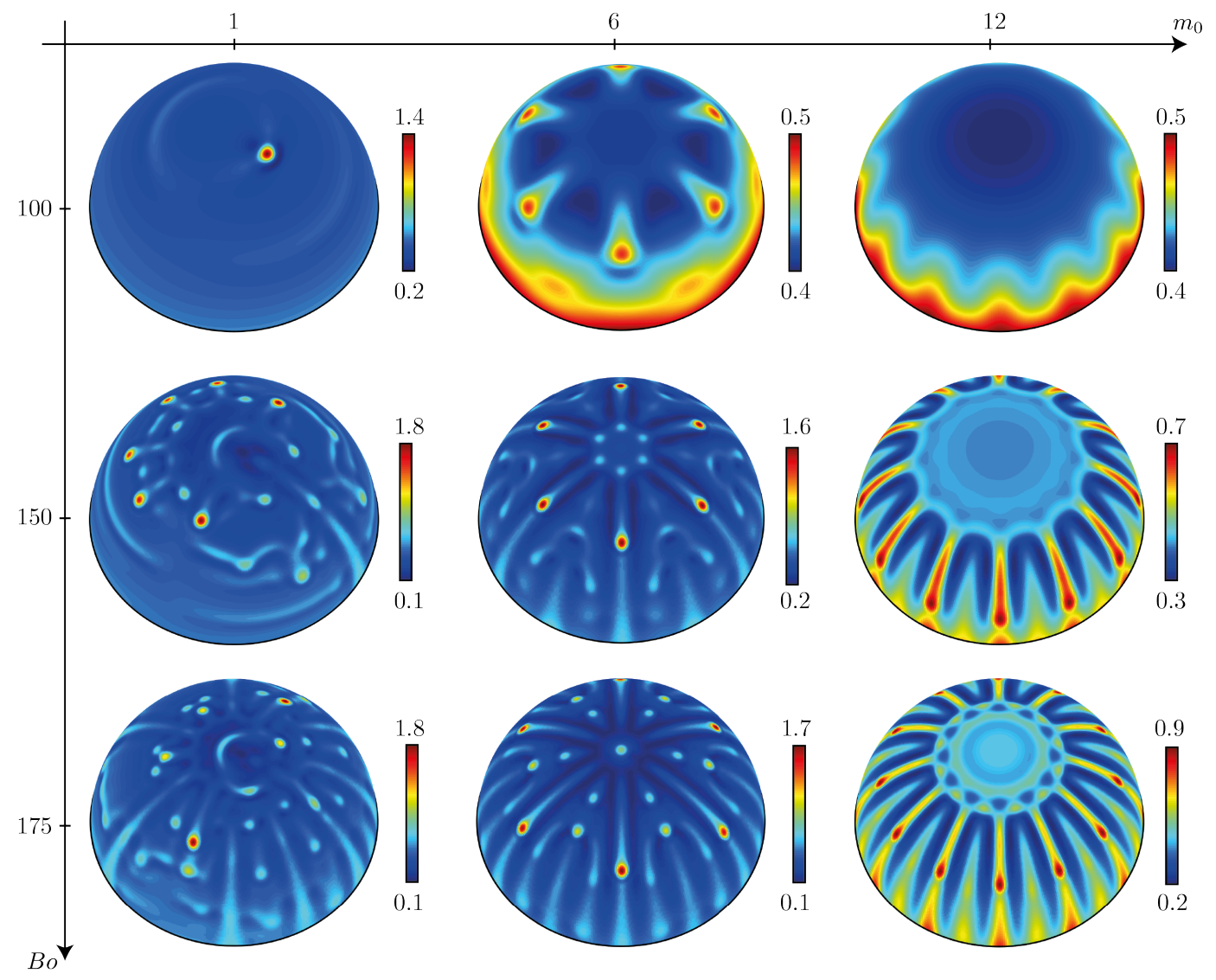

FIG. 11: Effect of the azimuthal wavenumber $m_{0}$ and of the Bond number $B o$ for the initial condition given by equation (15). $\varepsilon=10^{-2} ; m_{0}=1,6$ and $12 ; B o=100,150$ and

$$
175 ; t=4 \text {. }
$$

The perturbation dynamics for the different fundamental wavenumbers $m_{0}$ can be inferred by the spatio-temporal diagrams of the azimuthal disturbance norm $\mathcal{H}$ of Fig. 12 for $B o=$ 150. The most amplified azimuthal wavenumbers are shown in Fig. 13 and the evolution of the energy norm in Fig. 14. When $m_{0}=1$, the first droplet forms close to the north pole, but translates very fast along the substrate. While sliding, successive droplets form through the nonlinear interactions, as can be visible by the different ridges on Fig. 12. All successive harmonics $m=2,3, \ldots$ are formed (see Fig. 13). The energy is transferred from one harmonic 

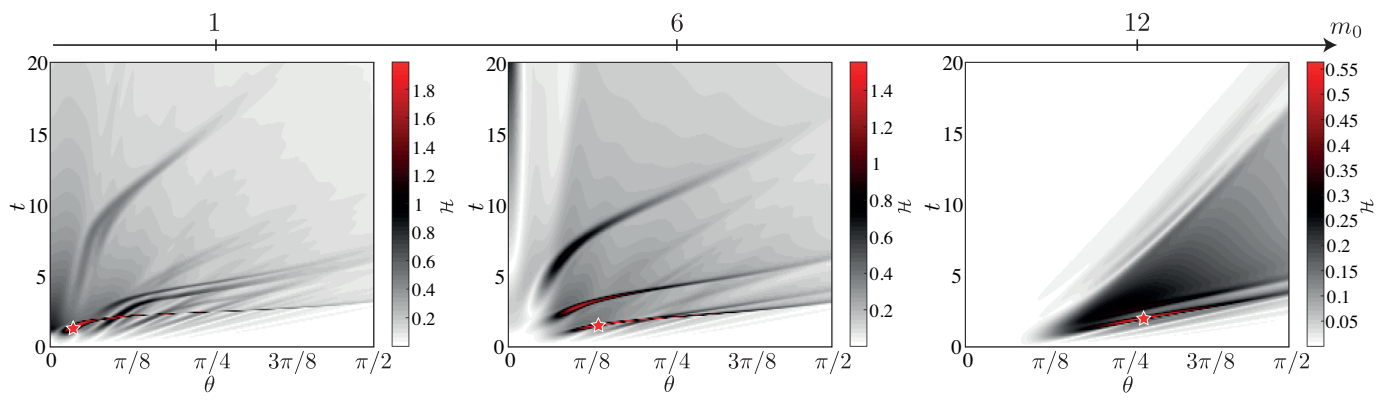

FIG. 12: Effect of the azimuthal wavenumber on the azimuthal disturbance norm $\mathcal{H}$ for $B o=150, \varepsilon=10^{-2}$ and $m_{0}=1,6$ and 12 with a linear optimal initial condition. The red star highlights the instant of largest amplitude.
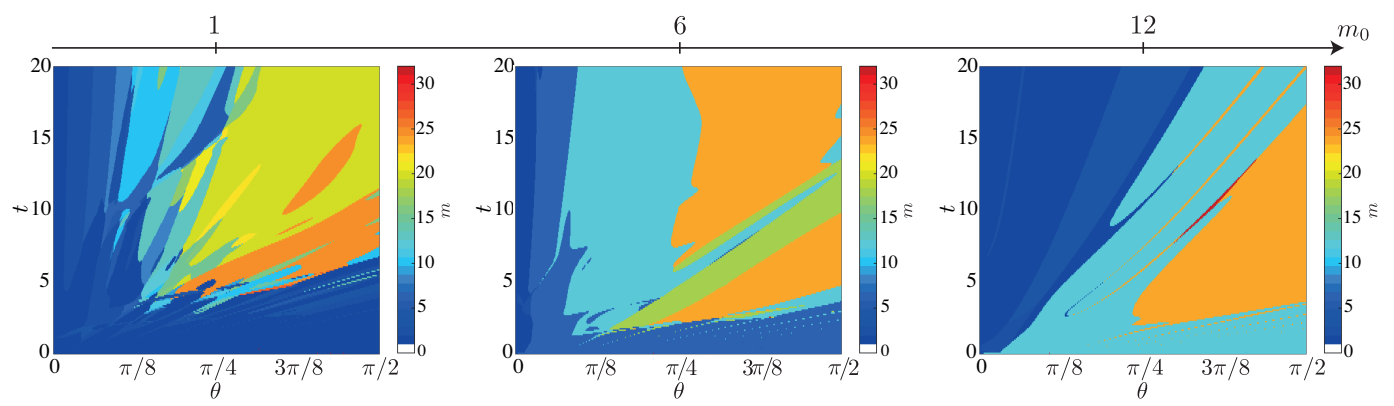

FIG. 13: Effect of the azimuthal wavenumber on the azimuthal mode $m$ with the largest amplitude for $B o=150, \varepsilon=10^{-2}$ and $m_{0}=1,6$ and 12 with a linear optimal initial condition. The colorbar indicates the $m$-values.

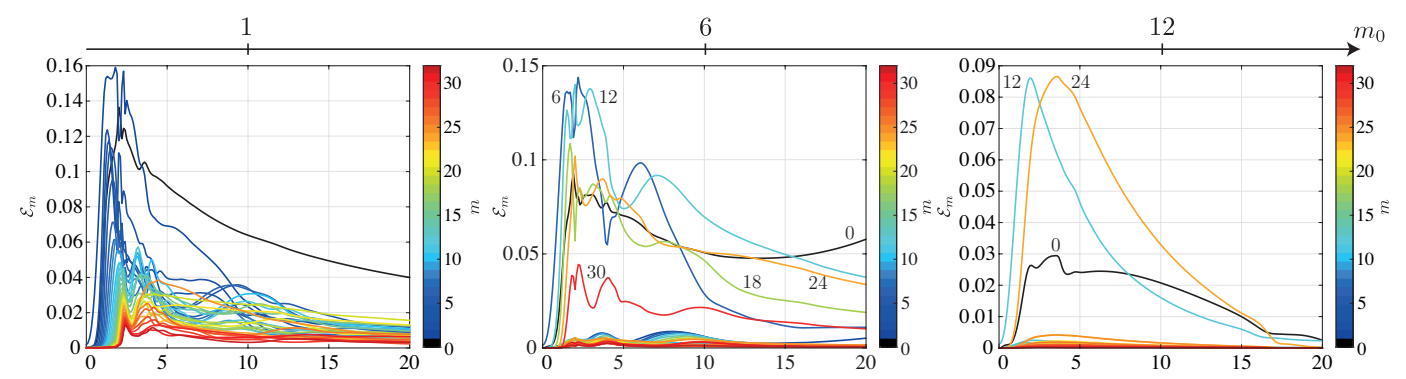

FIG. 14: Effect of the azimuthal wavenumber on the evolution of the energy norm $\mathcal{E}_{m}$ for $B o=150, \varepsilon=10^{-2}$ and $m_{0}=1,6$ and 12 with a linear optimal initial condition. The colorbar indicates the $m$-values.

to the successive one, while decreasing in amplitude (see Fig. 14). On the other hand, for $m_{0}=12$, the nonlinear dynamics is less rich. The first 12 perturbation peaks drain with an almost constant velocity, generating the harmonics $m=24$ (see Fig. 13) and modifying the mean film thickness (see Fig. 14). However, already for $t=20$, the disturbance amplitude is very small over the entire upper hemisphere. Higher order harmonics cannot be formed as they would correspond to too small wavelengths, which are stabilized by capillary forces. 


\section{Effect of the Bond number}

The film thickness maps at $t=4$ for $B o=100,150$ and 175 and the three azimuthal wavenumbers $m_{0}=1,6$ and 12 have been presented in Fig. 11. The spatio-temporal diagrams for the azimuthal disturbance norm $\mathcal{H}$ for $B o=150$ is shown in Figs. 6; qualitatively similar results have been obtained for $B o=175$. The spatio-temporal diagram of the disturbances for $B o=100$ can be inferred from Fig. 16(a). In agreement with the observations of the linear optimal growth study of Sec. III B, the disturbance amplitude increases with the Bond number. Due to the amplifying nature of the system, for a given initial disturbance amplitude $\varepsilon$, the linear growth might be sufficiently large, or not, for nonlinear effects to be relevant.

For $B o=100$, the linear growth is found to be insufficient to trigger strong nonlinear effects. The disturbance amplitude along the meridian $\phi=0$, where the thickest perturbation is located, presents ridges with almost a linear slope, evidencing the linearity of the dynamics, as will become clear in Sec. IV D (see Fig. 16(a)). Due to the absence of strong nonlinear interactions, the dominant azimuthal mode remains the fundamental one.

However, for $B o=150$ and $B o=175$, the linear growth is sufficiently large for the nonlinear effects to be relevant. The nonlinear interactions for $B o=175$ are stronger than for $B o=150$, resulting in an increased number of droplet generations (see Fig. 11). The nonlinear interactions at the pole are also stronger, resulting in a thicker droplet at $\theta=0$. As can be seen in Fig. 11 and in Fig. 15(a), the larger the Bond number, the smaller the polar angle where the most amplified azimuthal wavenumber changes from $m_{0}$ to $2 m_{0}$ and from $2 m_{0}$ to $4 m_{0}$.

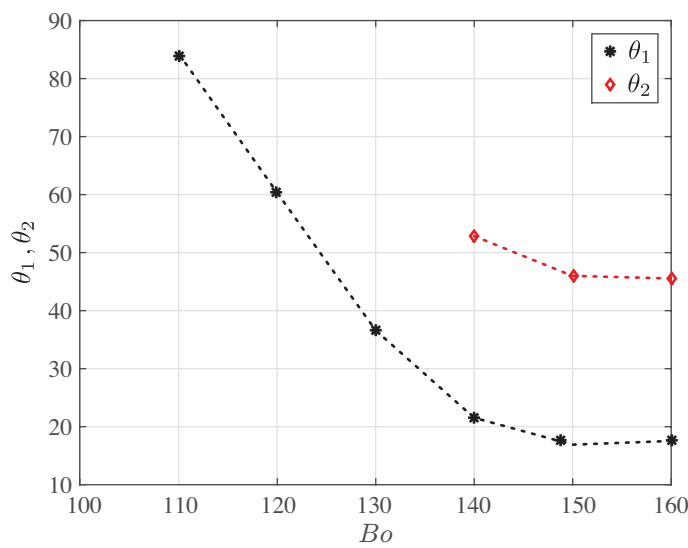

(a)

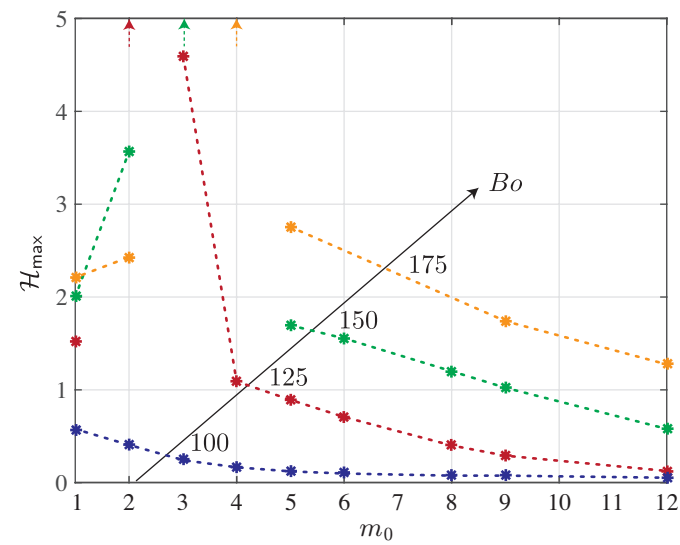

(b)

FIG. 15: (a) Effect of the Bond number on the angles $\theta_{1}$ and $\theta_{2}$ for the transition of the azimuthal mode with the largest amplitude from $m_{0}$ to $2 m_{0}$ and from $2 m_{0}$ to $4 m_{0}$, respectively, at $t=20$ (see Fig. 8). (b) Maximal azimuthal energy $\mathcal{H}_{\max }=\max _{(t, \theta)} \mathcal{H}$ as a function of the initial azimuthal wavenumber $m_{0}$ for $\varepsilon=10^{-2} ; B o=100,125,150$ and 175 with a linear optimal initial condition. Arrows for $m_{0}=2,3$ and 4 indicate that the largest azimuthal energy for $B o=125,150$ and 175 is larger than 5 , but cannot be computed as a thick droplet forms at the north pole and the simulations have to be interrupted. $\varepsilon=10^{-2}, m_{0}=6$ and $B o=100,110,120, \ldots, 160$ with a linear optimal initial condition. Dashed lines are added for visualization purposes.

By comparing the largest azimuthal disturbance norm $\mathcal{H}$ in time and space as a function of the fundamental wavenumber $m_{0}$ and the Bond number $B o$ we have found that for each Bond number there is an optimal $m_{0}$ for which the perturbation is the largest (see Fig. 15(b)). For $B o=100$ the largest azimuthal disturbance norm is reached for $m_{0}=1$ and monotonically decreases for greater $m_{0}$ values. For $B o=125$, the largest azimuthal disturbance norm is reached for $m_{0}=2$, whereas the optimal azimuthal wavenumber is 
$m_{0}=3$ or 4 for $B o=150$ and 175 . When the optimal azimuthal wavenumber is larger than 1, the most amplified pattern consists in a thick droplet at the pole formed through nonlinear interactions of the fundamentals, requiring the simulations to be interrupted once the droplet is too thick. Hence, the higher the Bond number, the larger the most amplified azimuthal wavenumber. The same conclusion was drawn for the polar wavenumber in Sec. III B.

The rationale for this result is that increasing the Bond number corresponds to decreasing the capillary length for a fixed initial film thickness $H_{i}^{*}$ and sphere radius $R$, or to increasing $H_{i}^{*} R$ for a fixed capillary length. As the most amplified wavelength of the classical RTI decreases with the capillary length ${ }^{14}$, it is not surprising that the typical spacing between the droplets is smaller when the Bond number increases. Consequently, more droplets can be located under a sphere of increased radius $R$.

\section{Pattern propagation velocity}

We have seen that the dynamics of the perturbations can be either linear or nonlinear. In the latter case the perturbations result in the formation of droplets which slide on the substrate. The advection velocity of the perturbation in the linear regime can be assumed to correspond to the free-surface velocity ${ }^{25,26}$. For a spherical substrate, the free-surface velocity of the smooth draining film $H$ is given by

$$
U=\frac{3}{2 \delta} \frac{Q}{H}=\frac{1}{2} \frac{1}{\delta} H^{2} \sin \theta,
$$

where $Q$ is the flux, given only by the gravity-induced drainage term $H^{3} \sin \theta / 3$. If one further assumes small angles $\theta$, the analytical solution of the draining flow $H(t)=1 / \sqrt{1+4 / 3 t}$ can be employed. The interfacial velocity for $t \gg 3 / 4$ becomes

$$
U \sim \frac{3}{8 \delta} \frac{\theta}{t} .
$$

Thus, the velocity of the free-surface is constant along the ray defined by $\theta / t=$ const. The exact free-surface velocity, Eq. (20), as well as the approximation for small angles, Eq. (21), are represented on the spatio-temporal diagram for $B o=100$ in Fig. 16(a). The isocontours of constant velocity are in good agreement with the ridges of the perturbations $\varepsilon h(\theta, \phi=0, t)$, confirming that small perturbations are advected by the free-surface velocity. For small $\theta$, the exact and asymptotic free-surface velocities are in good agreement. The asymptotic estimation (21) always underestimates the draining velocity (20) due to the assumption of small angles $\theta$, which results in a weaker tangential component of gravity.

For larger Bond numbers, the perturbations have larger amplitudes, the dynamics is nonlinear and the velocity of the formed droplets no longer corresponds to the free-surface velocity of the film. The thickness of the droplets is larger than the surrounding film, which increases their mobility and results in a larger sliding velocity. In order to quantify the velocity of thick droplets, it is instructive to consider them as one-dimensional shocks, for which the velocity is given by the Rankine-Hugoniot jump condition ${ }^{27}$

$$
s=\frac{\bar{Q}\left(\bar{H}_{L}\right)-\bar{Q}\left(\bar{H}_{r}\right)}{\bar{H}_{L}-\bar{H}_{R}} .
$$

As a shock we consider the downstream side of the sliding droplets, so that $\bar{H}_{L}$ is the largest thickness of the droplet and $\bar{H}_{R}$ is the smallest thickness just ahead of the droplet. The polar flux is (see also Eq. (1))

$$
\bar{Q}=\frac{1}{3}\left[\bar{H}^{3}\left(\frac{1}{B o} \bar{\kappa}_{\theta}+\delta \bar{H}_{\theta} \cos \theta+\sin \theta\right)\right],
$$




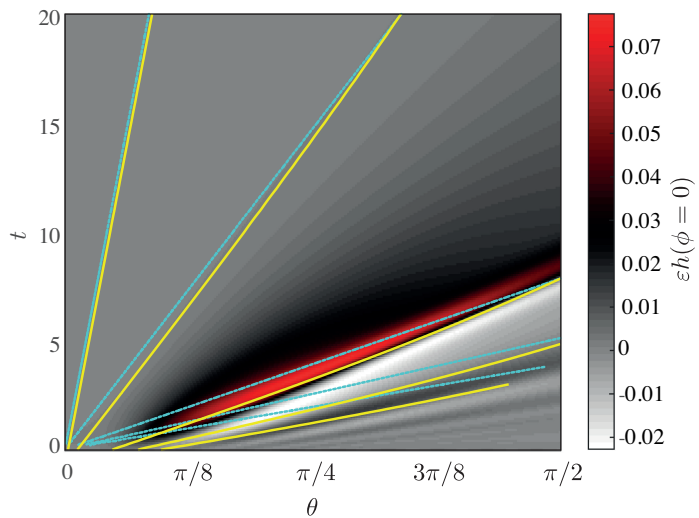

(a)

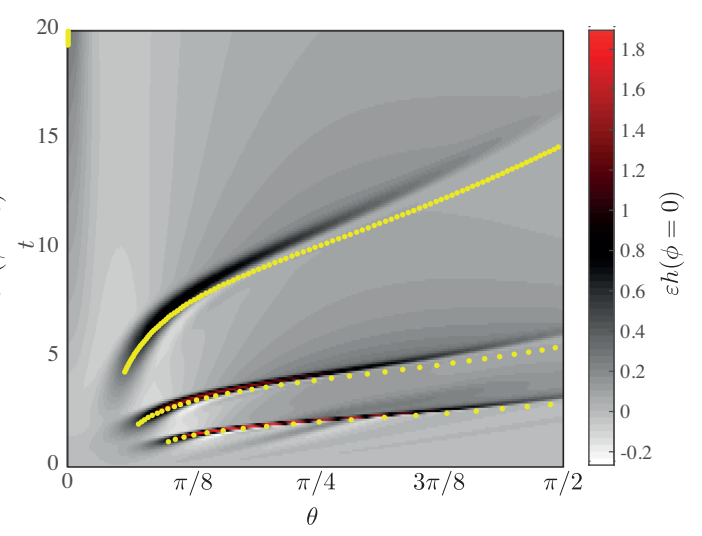

(b)

FIG. 16: (a) Spatio-temporal diagram of the film-thickness disturbance $\varepsilon h=\bar{H}-H$ at $\phi=0$ together with the isocontours of the free-surface velocity $U=0.1,0.4,1.5,2.3$ and 3 for the asymptotic solution (21) (turquoise dashed line) and for Eq. (20) with the numerical solution of the draining film (yellow solid line), respectively, for $B o=100$. (b) Spatio-temporal diagram of the film-thickness disturbance $\varepsilon h=\bar{H}-H$ at $\phi=0$ with the sliding-droplet trajectories obtained by the integration of the shock speed predicted by Eq. (22) (yellow circles) for the three droplet generations at $\phi=0$ for $B o=150$. For both panels, $m_{0}=6$ and $\varepsilon=10^{-2}$ with a linear optimal initial condition.

where the curvature is given by Eq. (2). Note that since the dynamics is nonlinear, the total film thickness $\bar{H}$ has to be considered. Although the sliding droplets are not discontinuous due to the surface-tension term in the flux and the fact that the problem is two-dimensional, the droplet velocity is well predicted by Eq. (22), in particular for the first generation of droplets, which are the thickest. To illustrate the good agreement we superpose the droplet trajectory obtained by integrating Eq. (22) in time on the spatio-temporal map of the film thickness disturbances $\varepsilon h(\theta, \phi=0, t)$ in Fig. 16(b). The velocity of thick droplets is therefore given by the ratio of the difference in fluxes and in film thicknesses at the droplet center and front. We have found that the velocity is not monotonous and is strongly affected by the transient nature of this flow. Furthermore, successive droplet generations have a sliding velocity larger than if they were flowing on a film thickness given by the draining solution, since previous drops have deposited a certain amount of fluid and have therefore increased the value of $\bar{H}_{R}$ (see Fig. 16(b)), resulting in an increased mobility. For the considered parameters of Fig. 16(b), the relative difference in velocities can go up to $20 \%$ for the second generation of droplets and up to $30 \%$ for the third generation.

\section{RANDOM NOISE INITIAL CONDITIONS}

To get closer to the experimental reality, a random white noise initial condition with uniform distribution is now considered. The same distribution of mean value 0 and range $2 \varepsilon$ is employed for all Bond numbers. As observable in the film-thickness maps of Fig. 17, where the same maximal initial disturbance amplitude $\varepsilon$ as in Fig. 11 is employed, no droplet forms for $B o=100$. The rationale for the different behaviour with respect to Fig. 11 is the nonoptimality of the random noise initial condition, which limits the nonlinear interactions. For the same reason, we observe only a thick droplet at $t=4$ for $B o=125$ and $B o=150$, whereas we have found in Sec. IV C that the most amplified mode for $B o=125$ should have an azimuthal wavenumber of 2 and 3 or 4 for $B o=150$, meaning that several droplets should form at short times. A single thick droplet would be the most amplified perturbation for $B o=100$ when the optimal initial condition (15) is considered. Therefore, the randomness of the initial condition shifts the value of Bond number for a given most 
amplified perturbation to larger values. For $B o=175$, the random initial condition gives rise to several droplets at short times, as one would expect for $B o=150$ when an optimal initial condition is considered.

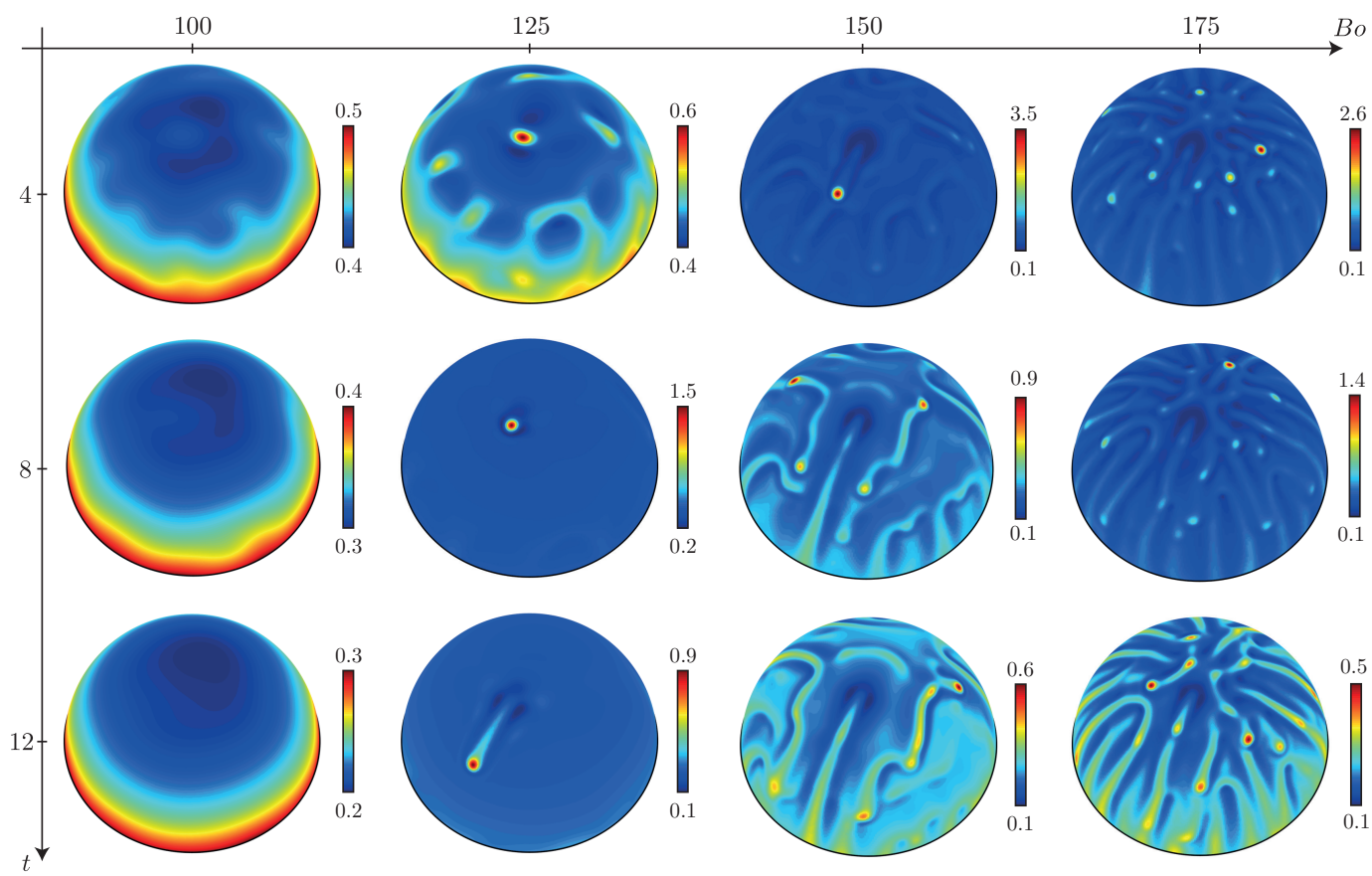

FIG. 17: Effect of the Bond number for a random noise initial condition with maximal amplitude $\varepsilon=10^{-2}, B o=100,125,150$ and 175 and $t=4,8$ and 12 . See Supplementary Movie $2^{28}$ for the entire evolution for $B o=175$.

Nonetheless, what we have learned from the nonlinear evolutions of the optimal initial conditions of Sec. IV also holds for the dynamics of a random noise initial condition. For example, the larger the Bond number, the faster the dynamics and the smaller the spacing between the droplets, as we have seen in Fig. 11 and Fig. 15. Furthermore, the quantities $\mathcal{H}, A_{m}$ and $\mathcal{E}_{m}$ can be employed to understand the dynamics. For $B o=100$, one recognizes the almost linear ridges in the spatio-temporal diagrams of Figs. 18 and 19, evidencing the linear dynamics as discussed for Fig. 16(a). From the evolution of the energy norm $\mathcal{E}_{m}$ it can be concluded that the initial energy, distributed over all modes because of the randomness of the spatial distribution, experiences only a weak transient growth for $B o=100$, where low-order modes reach the largest growths. For $B o=125$ and $B o=175$, the spatiotemporal diagrams of Fig. 18 highlight the formation of one and several droplets at short times, respectively. The larger the Bond number, the more the formed droplets, as found for the optimal initial conditions in Sec. IV C. The subsequent droplets generations are also visible. In particular, when a single drop is formed at short times, a cascade of droplets of the successive harmonics is generated as time increases, transferring energy from low to high azimuthal modes $m$ (see Fig. 20). By comparing the azimuthal modes with the largest amplitude (see Fig. 19), one confirms that the larger the Bond number, the wider the spectrum of the azimuthal wavenumbers $m$ of the perturbation.

Finally, the peculiar inverted-U shape perturbation is observed also for random initial conditions (see for instance Fig. 17 for $B o=175$ ). We believe that the reason for the formation of this general pattern is twofold. First, such perturbation structures form due to the drainage of two initially close perturbations which get apart while draining on the spherical geometry. Secondly, the trajectory of successive droplets strongly depends on the precise film thickness perturbations surrounding them. As can be seen in Figs. 5 and 17, as well as in the Supplementary Movies $1^{24}$ and $2^{28}$, a sliding drop leaves a wake behind, which 

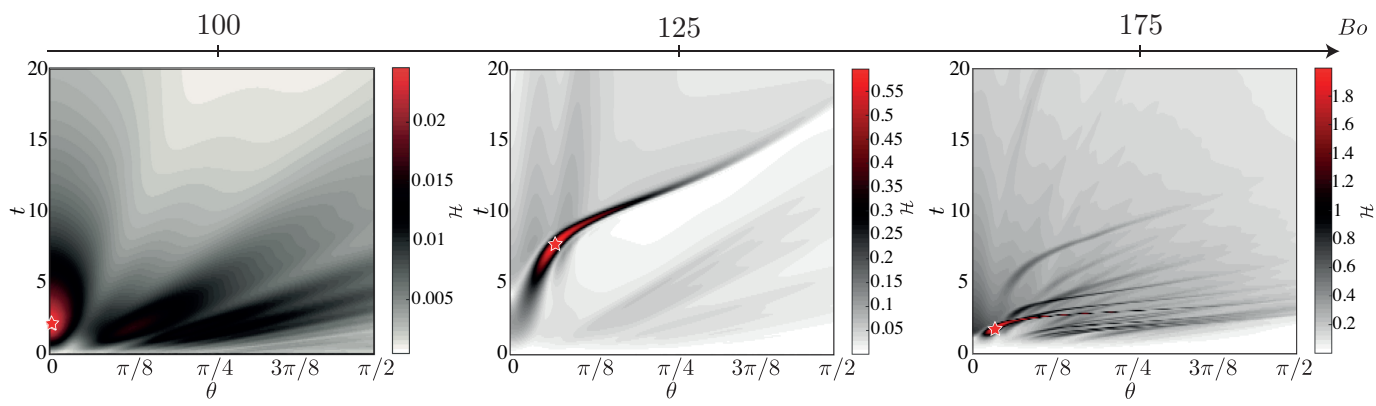

FIG. 18: Azimuthal disturbance norm $\mathcal{H}$ for a random noise initial condition with maximal amplitude $\varepsilon=10^{-2}$ and $B o=100,125$ and 175 . The red star highlights the instant of largest amplitude.

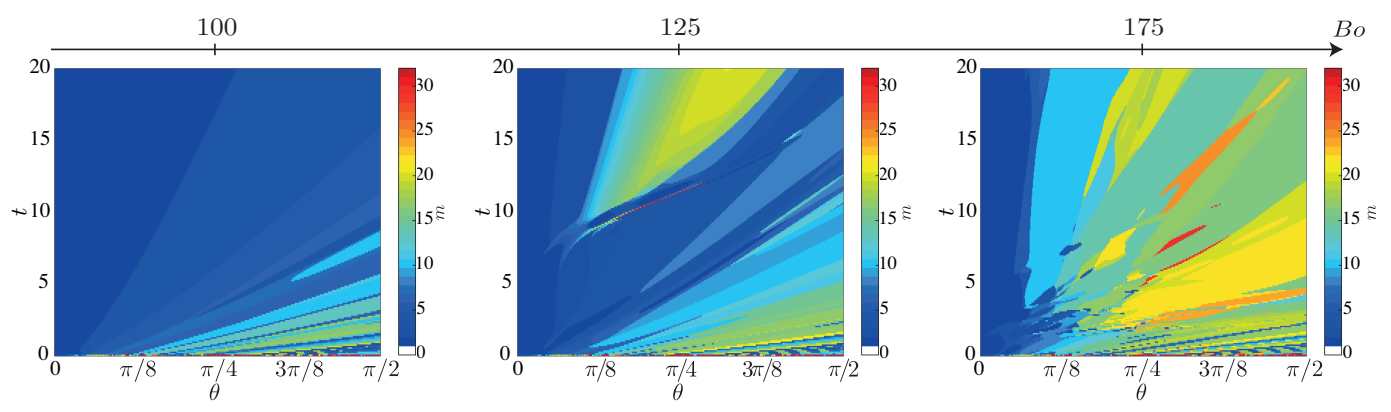

FIG. 19: Azimuthal mode $m$ with the largest amplitude for a random noise initial condition with maximal amplitude $\varepsilon=10^{-2}$ and $B o=100,125$ and 175. The colorbar indicates the $m$-values.

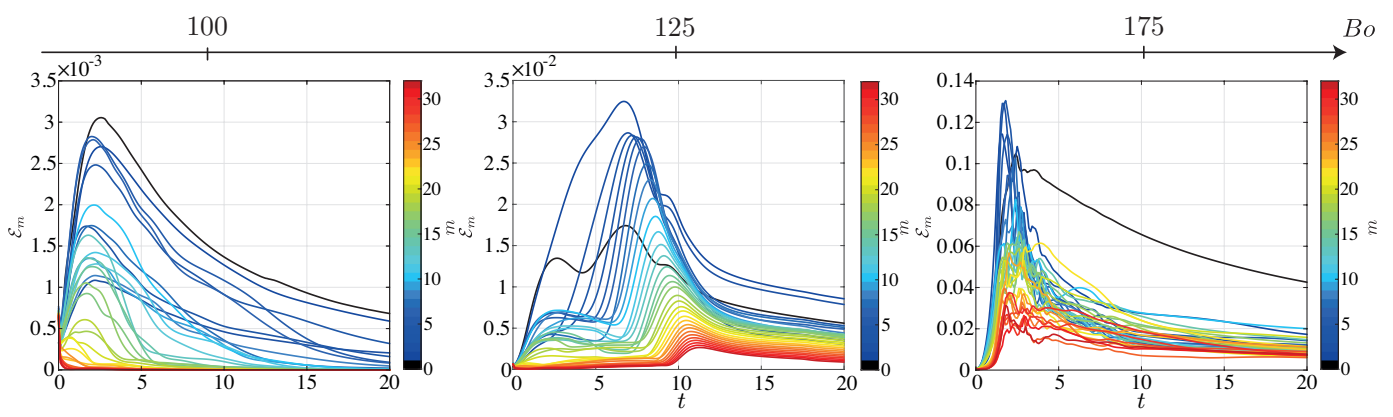

FIG. 20: Evolution of the energy norm $\mathcal{E}_{m}$ for for a random noise initial condition with maximal amplitude $\varepsilon=10^{-2}$ and $B o=100,125$ and 175 . The colorbar indicates the $m$-values.

is characterized by a reduced film thickness at its sides. Furthermore, it is also possible that a localized region of reduced film thickness exists due to the initial condition. Lister, Rallison, and Rees ${ }^{16}$ have shown that the anisotropic perturbation field induced by the motion of one droplet interacts with other perturbations and affects the droplet dynamics. In particular, a droplet is repelled by a region of reduced film thickness. Therefore, the thick film regions upstream of previously generated wakes or of zones of reduced film thickness drain in a way to minimize these repulsive forces. We conjecture that this is very likely to be at the origin of the inverted-U shape pattern. Only a droplet which is exactly aligned with the center of a previous wake can benefit from the thicker region at the center of the wake and follow the trajectory of previous droplets. 


\section{CONCLUSIONS}

The stability of a thin viscous film coating the interior of a sphere has been investigated. For a curved substrate, gravity has a twofold effect. On the one hand, it is the origin of the destabilization of the interface, through the component normal to it. On the other hand, the gravity component tangent to the substrate induces a flow which stabilizes the RayleighTaylor instability (RTI). The rationale for this stabilization mechanism is the thinning of the film and the stretching of the interface perturbations due to the drainage flow. The system is asymptotically stable and only a short-time algebraic growth can be achieved, when gravitational forces overcome the capillary forces by a given value. By performing a linear stability analysis for the critical region close to the north pole we have found that the Bond number for an initial growth of the perturbations is $B o=24$, the double of what is found for a cylindrical substrate ${ }^{17,21}$. The corresponding optimal wavenumber is identical to the classical RTI for a horizontal substrate, $k_{0}=\sqrt{B o / 2^{14}}$. The stabilizing effect of the curvature, firstly noted by Trinh et al. ${ }^{7}$ for a cylinder, is doubled as the mean curvature of a sphere is the double of the mean curvature of a cylinder. Physically speaking, the fluid drains over two main directions, rather than only along the polar direction of a cylinder. As a result, the thickness of the film close to the north pole decreases twice as fast as for a cylinder. The enhanced stabilization of the curvature explains why thin films do not destabilize under spherical substrates of radii larger than for cylinders, making it possible to fabricate large hollow chocolate eggs or elastic hemispherical shells of nearly uniform thickness ${ }^{8}$.

The linear optimal transient growth analysis yields an optimal polar wavenumber, but does not provide any optimal azimuthal wavenumber. The largest optimal disturbance amplitude increases exponentially with the Bond number, as for a cylindrical geometry, but has a smaller value. The exponential dependence with $B o$ confirms the amplifying nature of the system. Initial disturbances are amplified over a short time, by a Bondnumber-dependent factor. When the disturbance magnitude is of the order of the base flow, nonlinear effects become relevant. The critical Bond number for the transition to the nonlinear formation of droplets is found to be initial-disturbance amplitude dependent.

The nonlinear dynamics have been investigated by resolving the fully nonlinear lubrication equation over the entire geometry. The optimal initial conditions found by the linear analysis with a particular azimuthal wavenumber have been considered at first. We have found that there is an energy cascade from the fundamental azimuthal mode to the successive higher harmonics, until the azimuthal wavelength is too small and capillary forces stabilize the perturbation. At every polar location there is a dominant azimuthal wavenumber, increasing as the distance from the north pole, or the Bond number, increases. Furthermore, we have found that for each Bond number there is an optimal initial azimuthal wavenumber giving the largest nonlinear growth. The optimal initial azimuthal wavenumber is found to increase with the Bond number. Small-amplitude perturbations move with the free-surface velocity, whereas the velocity of thick droplets is well described by the one-dimensional shock theory.

Qualitatively similar results are found by resolving the nonlinear dynamics starting from an arbitrary random white noise initial condition. Depending on the Bond number, the following scenarios occur. For $B o<24$, the thin-film drains smoothly and no droplet forms. For moderate Bond numbers, yet sufficiently large for nonlinear effects to be important, a single droplet will form close to the pole. When sliding along the substrate, all successive harmonics will be excited and several droplets will appear at later times if the Bond number is sufficiently large. Instead, for very large Bond numbers, several droplets form close to the north pole already at short times. Nonlinear interactions will then promote the formation of their harmonics. The higher the Bond number, the larger the number of droplets generations, the closer they are to the pole and the faster the dynamics. Droplets form in the largest available space left by the previous ones, similarly to the formation of primordia in Phyllotaxic patterns ${ }^{29}$. At late times, the film thickness will present thicker regions, surrounded by narrow thin regions, corresponding to the wakes of the droplets. These structures resemble to the rivulets found for a cylindrical substrate ${ }^{18,30}$, yet with the 
significant difference that the rivulets in a sphere only form as a result of the droplet motion and are not an intrinsic instability pattern of the system.

If a thin film coats the outside of a spherical substrate, the interface is subjected to the Rayleigh-Taylor instability when it flows on the lower hemisphere. In this case the system is asymptotically unstable and no stable regime exists. The gravity-induced drainage is responsible for destabilizing the interface by focusing the flow at the south pole. At low Bond numbers a single droplet will form at the south pole, whereas a pattern of several droplets will form at high Bond numbers. Furthermore, we conjecture that rivulets will form at moderate Bond numbers.

It has to be stressed that the considered thin film is initially smooth. Otherwise, the moving capillary ridge close to the contact line would be unstable with respect to spanwise perturbations and would eventually result in the formation of fingers ${ }^{31,32}$, as investigated by Takagi and Huppert ${ }^{20}$ and Hammoud ${ }^{21}$. Thin liquid films coating spherical substrates can undergo also other types of instabilities when other forces are at play. For instance, if the liquid layer is submitted to an oscillating acceleration field, the liquid interface might become unstable to the Faraday instability ${ }^{33,34}$.

\section{ACKNOWLEDGMENTS}

The authors would like to acknowledge the valuable comments from the anonymous referees that helped to improve the manuscript. This work was funded by the ERC Grant No. SIMCOMICS 280117 and by the Swiss National Science Foundation Grant No. 200021_178971.

\section{Appendix A: Derivation of the lubrication equation}

The following description of the derivation of the equation is widely inspired by the one for the cylindrical geometry of Balestra et al. ${ }^{18}$. For a general derivation of the lubrication equation on curved substates see Refs. ${ }^{35-37}$. A thin film coating the interior of a sphere of radius $R$ is considered. The initial average thickness is $H_{i}^{*} \ll R$, yielding a small film aspect ratio $\delta=H_{i}^{*} / R \ll 1$. Using $H_{i}^{*}$ as characteristic length in the radial direction and $R$ as the characteristic length in the polar and azimuthal directions, the long wavelength approximation can be employed as $\delta \ll 1^{19}$. Mass conservation indicates that the velocity normal to the interface is much smaller than the polar and azimuthal components, $v^{*} \sim$ $\delta u^{*} \ll u^{*}$ and $v^{*} \sim \delta w^{*} \ll w^{*}$, respectively. Furthermore, the small Reynolds number for this flow allows the use of the Stokes equations and inertial effects can be neglected. The momentum equation in the radial direction is

$$
0=-\frac{1}{\rho} \frac{\partial p^{*}}{\partial r^{*}}-g \cos \theta
$$

and the boundary condition for the pressure is given by $p^{*}\left(R-\bar{H}^{*}, \theta, \phi\right)=p_{0}^{*}-\gamma \bar{\kappa}^{*}$, where $p_{0}^{*}$ is the external pressure, $\gamma$ the surface tension and $\bar{\kappa}^{*}$ the curvature of the interface. Equation (A1) can be integrated along the radial direction and using the boundary condition for the pressure, the pressure distribution $p^{*}\left(r^{*}, \theta, \phi\right)=p_{0}^{*}-\gamma \bar{\kappa}^{*}+\rho g \cos \theta\left(R-\bar{H}^{*}-r^{*}\right)$ is obtained. 
The polar and azimuthal components of the momentum equation read

$$
\begin{aligned}
0= & -\frac{1}{\rho r^{*}} \frac{\partial p^{*}}{\partial \theta}+\nu\left[\frac{1}{r^{* 2}} \frac{\partial}{\partial r^{*}}\left(r^{* 2} \frac{\partial u^{*}}{\partial r^{*}}\right)+\right. \\
& +\frac{1}{r^{* 2}} \frac{\partial}{\partial \theta}\left(\frac{1}{\sin \theta} \frac{\partial}{\partial \theta}\left(u^{*} \sin \theta\right)+\frac{1}{r^{* 2} \sin ^{2} \theta} \frac{\partial^{2} u^{*}}{\partial \phi^{* 2}}-\frac{2 \cot \theta}{r^{* 2} \sin \theta} \frac{\partial w^{*}}{\partial \phi}\right]+g \sin \theta, \\
0= & -\frac{1}{\rho r^{*} \sin \theta} \frac{\partial p^{*}}{\partial \phi}+\nu\left[\frac{1}{r^{* 2}} \frac{\partial}{\partial r^{*}}\left(r^{* 2} \frac{\partial w^{*}}{\partial r^{*}}\right)+\frac{1}{r^{* 2}} \frac{\partial}{\partial \theta}\left(\frac{1}{\sin \theta} \frac{\partial}{\partial \theta}\left(w^{*} \sin \theta\right)\right)+\right. \\
& \left.+\frac{1}{r^{* 2} \sin ^{2} \theta} \frac{\partial^{2} w^{*}}{\partial \phi^{2}}+\frac{2 \cot \theta}{r^{* 2} \sin \theta} \frac{\partial u^{*}}{\partial \phi}\right] .
\end{aligned}
$$

With the change of variable $r^{*}=R-s$, where $0 \leq s \leq \bar{H}^{*} \ll R$, the viscous terms in Eq. (A2) and Eq. (A3) reduce to $\nu \partial^{2} u^{*} / \partial s^{2}$ and $\nu \partial^{2} w^{*} / \partial s^{2}$, respectively, plus terms at least an order $\delta$ smaller. Considering only the dominant order of the viscous term, as well as the surface tension and gravitational terms, Eq. (A2) and Eq. (A3) with the expression for $p^{*}$ become:

$$
\begin{aligned}
& 0=\frac{\gamma \bar{\kappa}_{\theta}^{*}}{\rho R}+\frac{g \cos \theta \bar{H}_{\theta}^{*}}{R}+\nu \frac{\partial^{2} u^{*}}{\partial s^{2}}+g \sin \theta \\
& 0=\frac{\gamma \bar{\kappa}_{\phi}^{*}}{\rho R \sin \theta}+\frac{g \cos \theta \bar{H}_{\phi}^{*}}{R \sin \theta}+\nu \frac{\partial^{2} w^{*}}{\partial s^{2}}
\end{aligned}
$$

Equations (A4) and (A5) can be integrated twice and considering the zero-slip boundary condition at the sphere surface, $u^{*}(0, \theta, \phi)=0$ and $w^{*}(0, \theta, \phi)=0$, as well as the zero-shear stress interface, $\partial u^{*}\left(\bar{H}^{*}, \theta, \phi\right) / \partial s=0$ and $\partial w^{*}\left(\bar{H}^{*}, \theta, \phi\right) / \partial s=0$, the velocity components are found to be

$$
\begin{aligned}
u^{*}(s, \theta, \phi) & =\left(\frac{\gamma \bar{\kappa}_{\theta}^{*}}{\mu R}+\frac{\rho g \cos \theta \bar{H}_{\theta}^{*}}{\mu R}+\frac{\rho g \sin \theta}{\mu}\right)\left(\bar{H}^{*}-\frac{s}{2}\right) s, \\
w^{*}(s, \theta, \phi) & =\left(\frac{\gamma \bar{\kappa}_{\phi}^{*}}{\mu R \sin \theta}+\frac{\rho g \cos \theta \bar{H}_{\phi}^{*}}{\mu R \sin \theta}\right)\left(\bar{H}^{*}-\frac{s}{2}\right) s .
\end{aligned}
$$

The flow-rate in the polar direction is $Q^{\theta}(\theta, \phi)=\int_{0}^{\bar{H}^{*}} u^{*}(s, \theta, \phi) \mathrm{d} s$ and in the azimuthal direction is $Q^{\phi}(\theta, \phi)=\int_{0}^{\bar{H}^{*}} w^{*}(s, \theta, \phi) \mathrm{d} s$. Mass conservation in spherical coordinates $\partial \bar{H}^{*} / \partial t^{*}+(R \sin \theta)^{-1} \partial\left(\sin \theta Q^{\theta}\right) / \partial \theta+(R \sin \theta)^{-1} \partial Q^{\phi} / \partial \phi=0$ yields the lubrication equation:

$$
\begin{aligned}
\bar{H}_{t^{*}}^{*}+\frac{1}{3 \mu R \sin \theta}\left[\bar{H}^{* 3} \sin \theta(\underbrace{\frac{\gamma \bar{\kappa}_{\theta}^{*}}{R}}_{\mathbf{I}}+\underbrace{\frac{\rho g \cos \theta \bar{H}_{\theta}^{*}}{R}}_{\mathbf{I I}}+\underbrace{\rho g \sin \theta}_{\mathbf{I I I}})\right]_{\theta}+ \\
+\frac{1}{3 \mu R \sin \theta}\left[\frac{\bar{H}^{* 3}}{\sin \theta}(\underbrace{\frac{\gamma \bar{\kappa}_{\phi}^{*}}{R}}_{\mathbf{I}}+\underbrace{\frac{\rho g \cos \theta \bar{H}_{\phi}^{*}}{R}}_{\mathbf{I I}})\right]_{\phi}=0,
\end{aligned}
$$

The term $\mathbf{I}$ in the spatial variation of the flux corresponds to the surface tension effects, term II to the variation of the hydrostatic pressure distribution and term III to the drainage.

The free surface of the viscous film is defined by

$$
F\left(r^{*}, \theta, \phi, t^{*}\right)=r^{*}-\left[R-\bar{H}^{*}\left(\theta, \phi, t^{*}\right)\right]=0,
$$


its normal vector $\mathbf{n}$ is given by

$$
\mathbf{n}=\frac{\nabla F}{\|\nabla F\|}=\frac{\left[1, \frac{\bar{H}_{\theta}^{*}}{r^{*}}, \frac{\csc \theta \bar{H}_{\phi}^{*}}{r^{*}}\right]^{T}}{\sqrt{1+\left(\frac{\bar{H}_{\theta}^{*}}{r^{*}}\right)^{2}+\left(\frac{\csc \theta \bar{H}_{\phi}^{*}}{r^{*}}\right)^{2}}}
$$

at $r^{*}=R-\bar{H}^{*}$, and the interfacial curvature is $\bar{\kappa}^{*}=\nabla \cdot \mathbf{n}$.

Lengths can be nondimensionalized by the initial average film thickness $H_{i}^{*}$ and the time by the gravitational relaxation scale $\mu R /\left(\rho g H_{i}^{* 2}\right)$ so that the lubrication equation expressed with non-dimensional quantities finally reads:

$\bar{H}_{t}+\frac{1}{3 \sin \theta}\left[\bar{H}^{3} \sin \theta(\underbrace{\frac{1}{B o} \bar{\kappa}_{\theta}}_{\text {I }}+\underbrace{\delta \bar{H}_{\theta} \cos \theta}_{\text {II }}+\underbrace{\sin \theta}_{\text {III }})\right]_{\theta}+\frac{1}{3 \sin \theta}\left[\frac{\bar{H}^{3}}{\sin \theta}(\underbrace{\frac{1}{B o} \bar{\kappa}_{\phi}}_{\text {I }}+\underbrace{\delta \bar{H}_{\phi} \cos \theta}_{\text {II }})\right]_{\phi}=0$,

where $B o=\rho g H_{i}^{*} R / \gamma$ is the modified Bond number.

The curvature up to the second order in $\delta$ is :

$$
\bar{\kappa}=2 \delta+\delta^{2}\left(\bar{H}_{\theta \theta}+\cot \theta \bar{H}_{\theta}+\csc ^{2} \theta \bar{H}_{\phi \phi}+2 \bar{H}\right)+O\left(\delta^{3}\right) .
$$

\section{Appendix B: Derivation of the drainage solution}

If a uniform initial film thickness is assumed, $H(r, \phi, t=0)=1$, the lubrication equation at the pole (4) becomes

$$
\frac{\partial H}{\partial t}+\frac{1}{3 r} \frac{\partial}{\partial r}\left(H^{3} r^{2}\right)=0 \Leftrightarrow \frac{\partial H}{\partial t}+\frac{2}{3} H^{3}=0
$$

and the first order temporal solution, uniform in space, is $H(t)=\sqrt{1+4 t / 3^{20}}$. It can be noted that the thinning of the film is twice as fast as for a cylindrical substrate ${ }^{18}$.

The higher order solution for the drainage flow can be computed by considering a regular perturbation expansion of the film thickness starting from the equation (1) simplified for a uniform initial condition and under the assumption of small interface deformations

$$
\frac{\partial H}{\partial t}+\frac{1}{3 \sin \theta} \frac{\partial}{\partial \theta}\left(H^{3} \sin ^{2} \theta\right)=0
$$

As detailed in Ref. ${ }^{8}$, the asymptotic solution up to the fourth order is

$$
\begin{array}{r}
H(\theta, t) \approx \frac{1}{\sqrt{1+\frac{4}{3} t}}\left[1+\frac{\theta^{2}}{10}\left(1-\frac{1}{\left(1+\frac{4}{3} t\right)^{5 / 2}}\right)\right. \\
\left.+\theta^{4}\left(\frac{41}{4800}+\frac{9}{200\left(1+\frac{4}{3} t\right)^{5}}-\frac{1}{150\left(1+\frac{4}{3} t\right)^{5 / 2}}+\frac{135}{16\left(1+\frac{4}{3} t\right)^{4}}\right)\right] .
\end{array}
$$

\section{Appendix C: Numerical methods}

The two-dimensional nonlinear lubrication equation (1) is solved numerically using the finite element method of COMSOL Multiphysics. The total film thickness $\bar{H}$ as well as the total film curvature $\bar{\kappa}$ are the two variables. Quadratic elements with Lagrangian shape functions are employed on a free triangular grid of characteristic size 0.8 , which allows the consideration of azimuthal wavenumbers up to 78 . The relative tolerance for the timestepping algorithm is set to $10^{-3}$. The numerical procedure is validated with the analytical 
solution for the evolution of the draining thickness at the pole (see Fig. 21(a)) as well with the asymptotic solution for the spatial evolution of the drainage solution along the polar angle (see Fig. 21(b)). The largest relative error between the numerical and the analytical solution for the drainage at the pole is below $10^{-3}$.

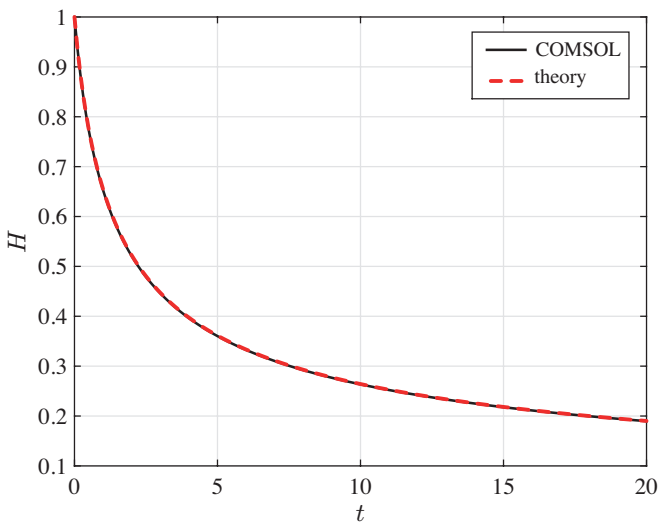

(a)

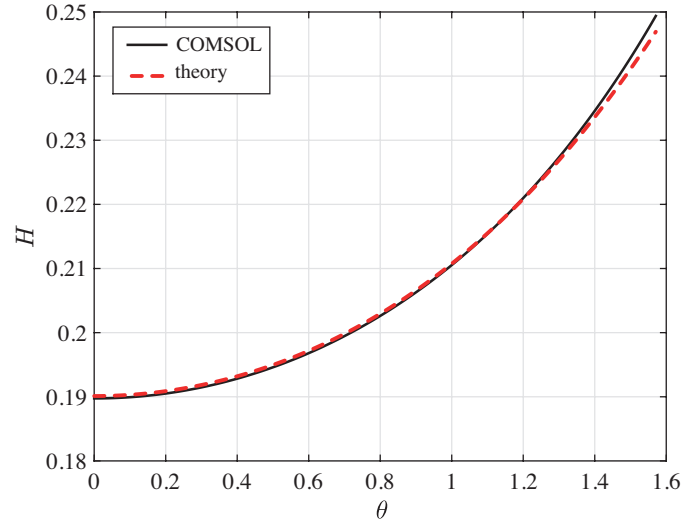

(b)

FIG. 21: Validation of the numerical scheme against the theoretical predictions. (a) Drainage at the pole, Eq. (B1), (b) drainage over the polar angle for $t=20$, Eq. (B3).

$$
B o=25 \text {. }
$$

\section{Appendix D: Alternative representation of the thin film evolution shown in Fig. 5}

For the sake of clarity, we show in Fig. 22 the corresponding the film thickness evolution shown in Fig. 5 using a constant colorbar range. One can clearly observe how the film thickness is reduced during time and how the successive droplet generations have a smaller amplitude, highlighting the transient nature of this flow.

${ }^{1}$ L. Rayleigh, "Investigation of the Character of the Equilibrium of an Incompressible Heavy Fluid of Variable Density," Proc. Lond. Math. Soc. s1-14, 170-177 (1882).

${ }^{2} \mathrm{G}$. Taylor, "The instability of liquid surfaces when accelerated in a direction perpendicular to their planes. I," Proc. R. Soc. Lond. A 201, 192-196 (1950).

${ }^{3}$ S. Chandrasekhar, Hydrodynamic and Hydromagnetic Stability (Dover, New York, 1981).

${ }^{4}$ D. H. Sharp, "An overview of Rayleigh-Taylor instability," Physica D 12, 3-18 (1984).

${ }^{5}$ S. G. Yiantsios and B. G. Higgins, "Rayleigh-Taylor instability in thin viscous films," Phys. Fluids A 1, 1484-1501 (1989).

${ }^{6} \mathrm{~L}$. Limat, "Instabilité d'un liquide suspendu sous un surplomb solide: Influence de l'épaisseur de la couche," Comptes rendus de l'Académie des sciences. Série 2, Mécanique, Physique, Chimie, Sciences de l'univers, Sciences de la Terre 317, 563-568 (1993).

${ }^{7}$ P. H. Trinh, H. Kim, N. Hammoud, P. D. Howell, S. J. Chapman, and H. A. Stone, "Curvature suppresses the Rayleigh-Taylor instability," Phys. Fluids 26, 051704 (2014).

${ }^{8}$ A. Lee, P.-T. Brun, J. Marthelot, G. Balestra, F. Gallaire, and P. M. Reis, "Fabrication of slender elastic shells by the coating of curved surfaces," Nat. Commun. 7, 11155 (2016).

${ }^{9}$ R. Krechetnikov, "Rayleigh-Taylor and Richtmyer-Meshkov instabilities of flat and curved interfaces," J. Fluid Mech. 625, 387-410 (2009).

${ }^{10}$ S. F. Kistler, P. M. Schweizer, et al., Liquid film coating (Springer, 1997).

${ }^{11}$ R. Kaita, L. Berzak, D. Boyle, T. Gray, E. Granstedt, G. Hammett, C. M. Jacobson, A. Jones, T. Kozub, H. Kugel, et al., "Experiments with liquid metal walls: Status of the lithium tokamak experiment," Fus. Eng. Des. 85, 874-881 (2010).

${ }^{12}$ R. Majeski, H. Kugel, R. Kaita, S. Avasarala, M. Bell, R. Bell, L. Berzak, P. Beiersdorfer, S. Gerhardt, E. Granstedt, et al., "The impact of lithium wall coatings on NSTX discharges and the engineering of the Lithium Tokamak eXperiment (LTX)," Fus. Eng. Des. 85, 1283-1289 (2010).

${ }^{13}$ F. Gallaire and P.-T. Brun, "Fluid dynamic instabilities: theory and application to pattern forming in complex media," Phil. Trans. R. Soc. A 375, 20160155 (2017).

${ }^{14} \mathrm{M}$. Fermigier, L. Limat, J. E. Wesfreid, P. Boudinet, and C. Quilliet, "Two-dimensional patterns in Rayleigh-Taylor instability of a thin layer," J. Fluid Mech. 236, 349-383 (1992). 

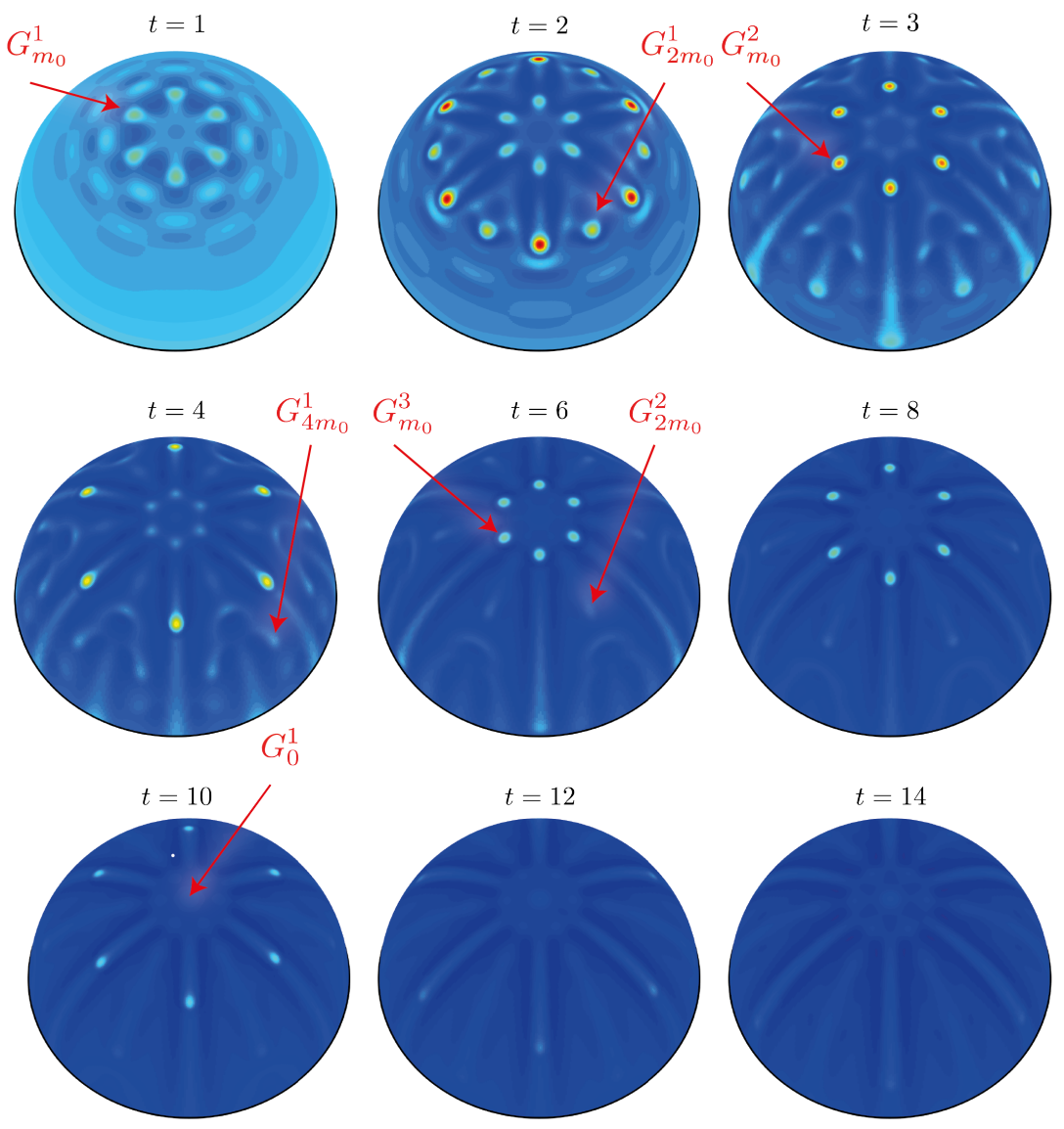

0

2.45

FIG. 22: Corresponding of Fig. 5 with a constant colorbar for the film thickness. Nonlinear evolution of the film thickness for the initial condition given by equation (15) for $B o=150, m_{0}=6$ and $\varepsilon=10^{-2}$ at the times $t=1,2,3,4,6,8,10,12$ and 14 . The droplet generations $G_{m_{0}}, G_{2 m_{0}}, G_{2 m_{0}}$ and $G_{0}$ are also highlighted.

${ }^{15}$ L. Limat, P. Jenffer, B. Dagens, E. Touron, M. Fermigier, and J. E. Wesfreid, "Gravitational instabilities of thin liquid layers: dynamics of pattern selection," Physica D 61, 166-182 (1992).

${ }^{16}$ J. R. Lister, J. M. Rallison, and S. J. Rees, "The nonlinear dynamics of pendent drops on a thin film coating the underside of a ceiling," J. Fluid Mech. 647, 239-264 (2010).

${ }^{17}$ G. Balestra, P.-T. Brun, and F. Gallaire, "Rayleigh-Taylor instability under curved substrates: An optimal transient growth analysis," Phys. Rev. Fluids 1, 083902 (2016).

${ }^{18}$ G. Balestra, N. Kofman, P.-T. Brun, B. Scheid, and F. Gallaire, "Three-dimensional Rayleigh-Taylor instability under a unidirectional curved substrate," J. Fluid Mech. 837, 19-47 (2018).

${ }^{19}$ A. Oron, S. H. Davis, and S. G. Bankoff, "Long-scale evolution of thin liquid films," Rev. Mod. Phys. 69, 931 (1997)

${ }^{20}$ D. Takagi and H. E. Huppert, "Flow and instability of thin films on a cylinder and sphere," J. Fluid Mech. 647, 221 (2010).

${ }^{21}$ N. Hammoud, On Instabilities in Thin-Film Flows, Ph.D. thesis, Princeton University (2016).

${ }^{22}$ J. Eggers and E. Villermaux, "Physics of liquid jets," Rep. Prog. Phys. 71, 036601 (2008).

${ }^{23}$ A. Indeikina, I. Veretennikov, and H.-C. Chang, "Drop fall-off from pendent rivulets," J. Fluid Mech. 338, 173-201 (1997).

24 "See supplemental material at [url will be inserted by publisher] for the movie of the film thickness evolution of fig. 5." ().

${ }^{25}$ P.-T. Brun, A. Damiano, P. Rieu, G. Balestra, and F. Gallaire, "Rayleigh-Taylor instability under an inclined plane," Phys. Fluids 27, 084107 (2015).

${ }^{26} \mathrm{~B}$. Scheid, N. Kofman, and W. Rohlfs, "Critical inclination for absolute/convective instability transition in inverted falling films," Phys. Fluids 28, 044107 (2016). 
${ }^{27}$ R. J. LeVeque, "Conservative methods for nonlinear problems," in Numerical Methods for Conservation Laws (Birkhäuser Basel, Basel, 1990) pp. 122-135.

28 "See supplemental material at [url will be inserted by publisher] for the movie of the film thickness evolution of fig. 17." ().

${ }^{29}$ S. Douady and Y. Couder, "Phyllotaxis as a dynamical self organizing process. Part I: the spiral modes resulting from time-periodic iterations," J. Theor. Biol. 178, 255-273 (1996).

${ }^{30}$ M. Rietz, B. Scheid, F. Gallaire, N. Kofman, R. Kneer, and W. Rohlfs, "Dynamics of falling films on the outside of a vertical rotating cylinder: waves, rivulets and dripping transitions," J. Fluid Mech. 832, 189-211 (2017).

${ }^{31}$ N. Fraysse and G. M. Homsy, "An experimental study of rivulet instabilities in centrifugal spin coating of viscous Newtonian and non-Newtonian fluids," Phys. Fluids 6, 1491-1504 (1994).

${ }^{32}$ T.-S. Lin, L. Kondic, and A. Filippov, "Thin films flowing down inverted substrates: Three-dimensional flow," Phys. Fluids 24, 022105 (2012).

${ }^{33}$ C. Falcon, E. Falcon, U. Bortolozzo, and S. Fauve, "Capillary wave turbulence on a spherical fluid surface in low gravity," EPL (Europhysics Letters) 86, 14002 (2009).

${ }^{34}$ A.-h. E. Adou and L. S. Tuckerman, "Faraday instability on a sphere: Floquet analysis," J. Fluid Mech. 805, 591-610 (2016).

${ }^{35}$ L. Schwartz and D. Weidner, "Modeling of coating flows on curved surfaces," J. Eng. Math. 29, 91-103 (1995).

${ }^{36}$ R. V. Roy, A. Roberts, and M. Simpson, "A lubrication model of coating flows over a curved substrate in space," J. Fluid Mech. 454, 235-261 (2002).

${ }^{37}$ P. Howell, "Surface-tension-driven flow on a moving curved surface," J. Eng. Math. 45, 283-308 (2003). 